SUPPORTING INFORMATION

\title{
Catalytic Asymmetric Synthesis of Phthioceranic Acid, a Heptamethyl-Branched Acid from Mycobacterium tuberculosis
}

Bjorn ter Horst, Ben L. Feringa* and Adriaan J.

Minnaard*

Department of Organic and Molecular Inorganic Chemistry, Stratingh Institute for Chemistry, University of Groningen, Nijenborgh 4, 9747 AG, Groningen, The Netherlands 


\section{General Procedures:}

All reactions were carried out under nitrogen atmosphere using dried glassware. All solvents were dried and distilled before use according to standard procedures. $t$-BuOMe was purchased as anhydrous grade, stored on $4 \AA \mathrm{MS}$ and used without further purification. Ligand 4 was generously provided by Solvias. $\mathrm{CuBr} \bullet \mathrm{SMe}_{2}$ was purchased from Aldrich or Acros and used without further purification. Grignard reagent $\mathrm{MeMgBr}$ was purchased from Aldrich. Grignard reagents were titrated using $s-\mathrm{BuOH}$ and catalytic amounts of 1,10-phenanthroline.

Chromatography: Merck silica gel type 9385 230-400 mesh, TLC: Merck silica gel 60, $0.25 \mathrm{~mm}$. Components were visualized by staining with Seebach's reagent: a mixture of phosphomolybdic acid $(25 \mathrm{~g})$, cerium (IV) sulfate $(7.5 \mathrm{~g}), \mathrm{H}_{2} \mathrm{O}(500 \mathrm{~mL})$ and $\mathrm{H}_{2} \mathrm{SO}_{4}(25 \mathrm{~mL})$.

Mass spectra were recorded on a AEI-MS-902 mass spectrometer. ${ }^{1} \mathrm{H}$ - and ${ }^{13} \mathrm{C}-\mathrm{NMR}$ spectra were recorded on a Varian AMX400 $\left(400,100.59 \mathrm{MHz}\right.$, respectively) using $\mathrm{CDCl}_{3}$ as solvent. Chemical shift values are reported in ppm with the solvent resonance as the internal standard $\left(\mathrm{CHCl}_{3}: \delta 7.26\right.$ for ${ }^{1} \mathrm{H}, \delta 77.0$ for ${ }^{13} \mathrm{C}$ ). Data are reported as follows: chemical shifts, multiplicity $(\mathrm{s}=$ singlet, $\mathrm{d}=$ doublet, $\mathrm{t}=$ triplet, $\mathrm{q}=$ quartet, $\mathrm{br}=$ broad, $\mathrm{m}=$ multiplet), coupling constants $(\mathrm{Hz})$, and integration. Optical rotations were measured on a Schmidt + Haensch polarimeter (Polartronic MH8) with a $10 \mathrm{~cm}$ cell ( $c$ given in $\mathrm{g} / 100 \mathrm{~mL}$ ). Enantiomeric excess was determined by capillary GC analysis (Chiraldex A-TA column (30 m x $0.25 \mathrm{~mm}$ ) using a flame ionization detector and compared with racemic products. 
Experimental:

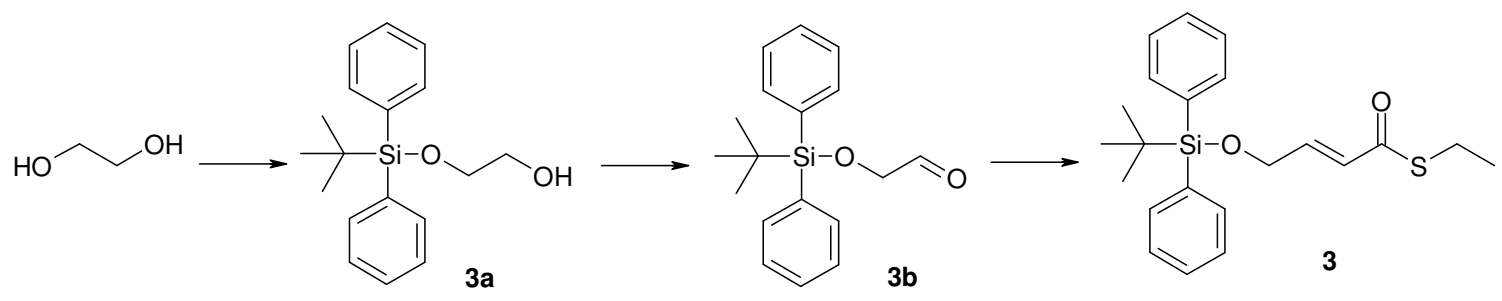

(E)-4-(tert-Butyl-diphenyl-silanyloxy)-but-2-enethioic acid S-ethyl ester (3)

tert-Butyl-chloro-diphenyl-silane $(10.0 \mathrm{~mL}, 38.5 \mathrm{mmol})$ was added to a stirred solution of 6 equiv of ethane-1,2-diol (12.0 mL, $231 \mathrm{mmol})$ and 1.1 equiv of imidazole $(2.88 \mathrm{~g}, 42.4 \mathrm{mmol})$ in $200 \mathrm{~mL}$ of THF under nitrogen atmosphere. The resulting mixture was stirred for $24 \mathrm{~h}$ at $\mathrm{rt}$ and quenched with $200 \mathrm{~mL}$ water followed by addition of $200 \mathrm{~mL}$ of diethyl ether. After phase separation and extraction of the aqueous phase with 3 portions of $200 \mathrm{~mL}$ of diethyl ether, the combined organic phases were dried over $\mathrm{MgSO}_{4}$, concentrated under reduced pressure and purified by flash chromatography (eluent pentane/EtOAc 4:1) to afford 3a as a colorless oil (9.14 g, 79\% yield). A solution of 3a (9.14 g, $30.5 \mathrm{mmol})$ and 1.3 equiv of iodoxybenzoic acid (IBX) (11.09 g, $39.61 \mathrm{mmol})$ in $200 \mathrm{~mL}$ of EtOAc was refluxed for $24 \mathrm{~h}$ and cooled to rt. IBX and benzoic acid were filtered off through Celite path and washed with EtOAc. The filtrate was concentrated under reduced pressure to give the aldehyde $\mathbf{3 b}(8.81 \mathrm{~g}, 97 \%$ yield $)$ which was used in the next step without purification. A solution of $\mathbf{3 b}(8.81 \mathrm{~g}, 29.6 \mathrm{mmol})$ and $\mathrm{Ph}_{3} \mathrm{PCHCOSEt}(13.99 \mathrm{~g}, 38.42$ $\mathrm{mmol})$ in $\mathrm{CH}_{2} \mathrm{Cl}_{2}(150 \mathrm{~mL})$ was refluxed for $24 \mathrm{~h}$. The solution was concentrated under reduced pressure and purified by flash chromatography (eluent pentane/ether 40:1) to afford $\alpha, \beta$-unsaturated thioester $\mathbf{3}$ as a colourless oil (9.078 g, 80\% yield). ${ }^{1} \mathrm{H}-\mathrm{NMR}\left(400 \mathrm{MHz}, \mathrm{CDCl}_{3}\right): \delta 7.67(\mathrm{dd}, J=7.6,1.2 \mathrm{~Hz}, 4 \mathrm{H}), 7.42(\mathrm{~m}$, $6 \mathrm{H}), 6.90(\mathrm{dt}, J=15.2,3.2 \mathrm{~Hz}, 1 \mathrm{H}), 6.55(\mathrm{dt}, J=14.8,2.4 \mathrm{~Hz}, 1 \mathrm{H}), 4.35(\mathrm{~m}, 2 \mathrm{H}), 2.98(\mathrm{q}, J=7.6 \mathrm{~Hz}, 2 \mathrm{H})$, $1.31(\mathrm{t}, J=7.2 \mathrm{~Hz}, 3 \mathrm{H}), 1.09$ (s, 9H). ${ }^{13} \mathrm{C}-\mathrm{NMR}\left(100.6 \mathrm{MHz}, \mathrm{CDCl}_{3}\right): \delta 190.07$ (s), $142.70(\mathrm{~d}), 135.40(\mathrm{~d})$, $132.86(\mathrm{~s}), 129.86(\mathrm{~d}), 127.80$ (d), 126.73 (d), 62.77 (t), 26.74 (q), 23.18 (t), 19.24 (s), 14.76 (q). MS(EI+) for $\mathrm{C}_{22} \mathrm{H}_{28} \mathrm{O}_{2} \mathrm{SSi}: \mathrm{m} / \mathrm{z}(\%)=327$ (71\%, M - $t$-Butyl), $384(100 \%, M)$. HRMS(EI+) for $\mathrm{C}_{22} \mathrm{H}_{28} \mathrm{O}_{2} \mathrm{SSi}: \mathrm{m} / \mathrm{z}(\%)$ $=327(100 \%, \mathrm{M}-t$-Butyl), Measured Mass: 327.0875 Da, Calculated Mass: 327.0875 Da. For spectral data see: Chem. Commun., 2007, 489-491.

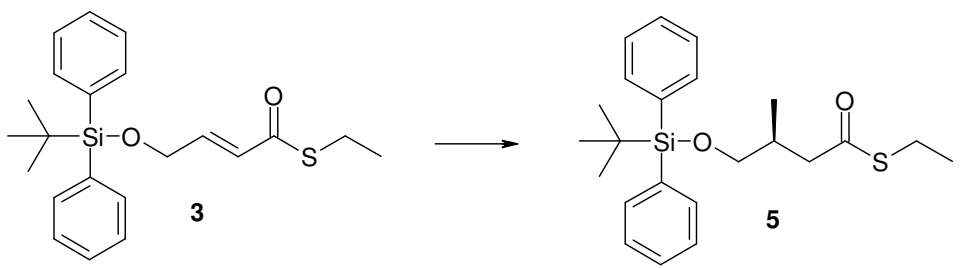

(-)-(S)-4-(tert-Butyl-diphenyl-silanyloxy)-3-methyl-thiobutyric acid S-ethyl ester (5)

$\left(R, S_{F e}\right)$-Josiphos (4) $\cdot \mathrm{CuBr}$ complex $(58 \mathrm{mg}, 0.078 \mathrm{mmol}, 1 \mathrm{~mol} \%)$ was dissolved in $t$-BuOMe $(48 \mathrm{~mL})$ under nitrogen. The solution was cooled to $-75^{\circ} \mathrm{C}$ and methylmagnesium bromide $(9.37 \mathrm{mmol}$, solution in diethyl ether) was added dropwise over $10 \mathrm{~min}$. After stirring for $10 \mathrm{~min}$, a solution of thioester 3 (3.0 g, $7.81 \mathrm{mmol})$ in $t$-BuOMe $(13.6 \mathrm{~mL})$ was added via syringe pump over $1 \mathrm{~h}$. The reaction mixture was stirred at $-75{ }^{\circ} \mathrm{C}$ for $17 \mathrm{~h}$, then quenched by the addition of $\mathrm{MeOH}$ and allowed to warm to room temperature. Saturated aqueous $\mathrm{NH}_{4} \mathrm{Cl}$ solution was then added. After phase separation and extraction of the aqueous phase with 3 portions of diethyl ether $(50 \mathrm{~mL})$, the combined organic phases were dried over $\mathrm{MgSO}_{4}$, concentrated under reduced pressure and purified by flash chromatography (eluent pentane/ether 40:1) to afford 5 as a colourless oil $\left(2.968 \mathrm{~g}, 95 \%\right.$ yield, $98 \%$ ee, $\left.[\alpha]_{\mathrm{D}}=-8.0\left(c=1.2, \mathrm{CHCl}_{3}\right)\right) .{ }^{1} \mathrm{H}-\mathrm{NMR}(400 \mathrm{MHz}$, $\left.\mathrm{CDCl}_{3}\right): \delta 7.66(\mathrm{dd}, J=6.8,1.4 \mathrm{~Hz}, 4 \mathrm{H}), 7.41(\mathrm{~m}, 6 \mathrm{H}), 3.55(\mathrm{dd}, J=10.0,5.3 \mathrm{~Hz}, 1 \mathrm{H}), 3.46(\mathrm{dd}, J=9.9$, $6.3 \mathrm{~Hz}, 1 \mathrm{H}), 2.88(\mathrm{q}, J=7.4 \mathrm{~Hz}, 2 \mathrm{H}), 2.83(\mathrm{dd}, J=14.5,5.3 \mathrm{~Hz}, 1 \mathrm{H}), 2.38(\mathrm{dd}, J=14.5,8.4 \mathrm{~Hz}, 1 \mathrm{H}), 2.28$ $(\mathrm{m}, 1 \mathrm{H}), 1.25(\mathrm{t}, J=7.4 \mathrm{~Hz}, 3 \mathrm{H}), 1.15(\mathrm{~s}, 9 \mathrm{H}), 0.98(\mathrm{~d}, J=6.6 \mathrm{~Hz}, 3 \mathrm{H}) .{ }^{13} \mathrm{C}-\mathrm{NMR}\left(100.6 \mathrm{MHz}, \mathrm{CDCl}_{3}\right)$ : $199.08(\mathrm{~s}), 135.57(\mathrm{~d}), 133.63(\mathrm{~s}), 129.58(\mathrm{~d}), 127.50(\mathrm{~d}), 67.90(\mathrm{t}), 47.75(\mathrm{t}), 33.76(\mathrm{~d}), 26.84(\mathrm{q}), 23.27(\mathrm{t})$, 19.28 (s), 16.40 (q), 14.77 (q). MS(EI+) for $\mathrm{C}_{23} \mathrm{H}_{32} \mathrm{O}_{2} \mathrm{SSi}: \mathrm{m} / \mathrm{z}(\%)=343(100 \%, \mathrm{M}-t$-Butyl), MS(CI+) for $\mathrm{C}_{23} \mathrm{H}_{32} \mathrm{O}_{2} \mathrm{SSi}: \mathrm{m} / \mathrm{z}(\%)=418\left(37.5 \%, \mathrm{M}+\mathrm{NH}_{4}^{+}\right), 401\left(100 \%, \mathrm{M}+\mathrm{H}^{+}\right)$. HRMS(EI+) for $\mathrm{C}_{23} \mathrm{H}_{32} \mathrm{O}_{2} \mathrm{SSi}:$ $\mathrm{m} / \mathrm{z}(\%)=343(100 \%, \mathrm{M}-t$-Butyl), Measured Mass: 343.1183 Da, Calculated Mass: 343.1188 Da. For spectral data see: Chem. Commun., 2007, 489-491. 
E.e. and absolute configuration were determined by removal of the tert-butyldiphenylsilyl group from 5a, prepared with $\left(S, R_{F e}\right)$-Josiphos, resulting in lactone $\mathbf{5 b}$. The absolute configuration of lactone $\mathbf{5 b}$ has been previously reported ${ }^{1,2,3}$ Lit: $[\alpha]_{\mathrm{D}}=-24.7(c=1.7, \mathrm{MeOH})$ for the $S$ - configuration. Found: $[\alpha]_{\mathrm{D}}=+21.6(c$ $=0.5, \mathrm{MeOH})$. Determination of enantiomeric excess was achieved by GC analysis [Chiraldex AT-A (30.0 $\mathrm{m} \times 0.25 \mathrm{~mm}$ ), $1.0 \mathrm{ml} \mathrm{min}{ }^{-1}$, initial temp. $50{ }^{\circ} \mathrm{C}$ then $5{ }^{\circ} \mathrm{C} \mathrm{min}^{-1}$ to final temp. $170{ }^{\circ} \mathrm{C}, 19.7 \mathrm{~min}$ (minor), 19.9 (major) shows $98 \%$ ee.]
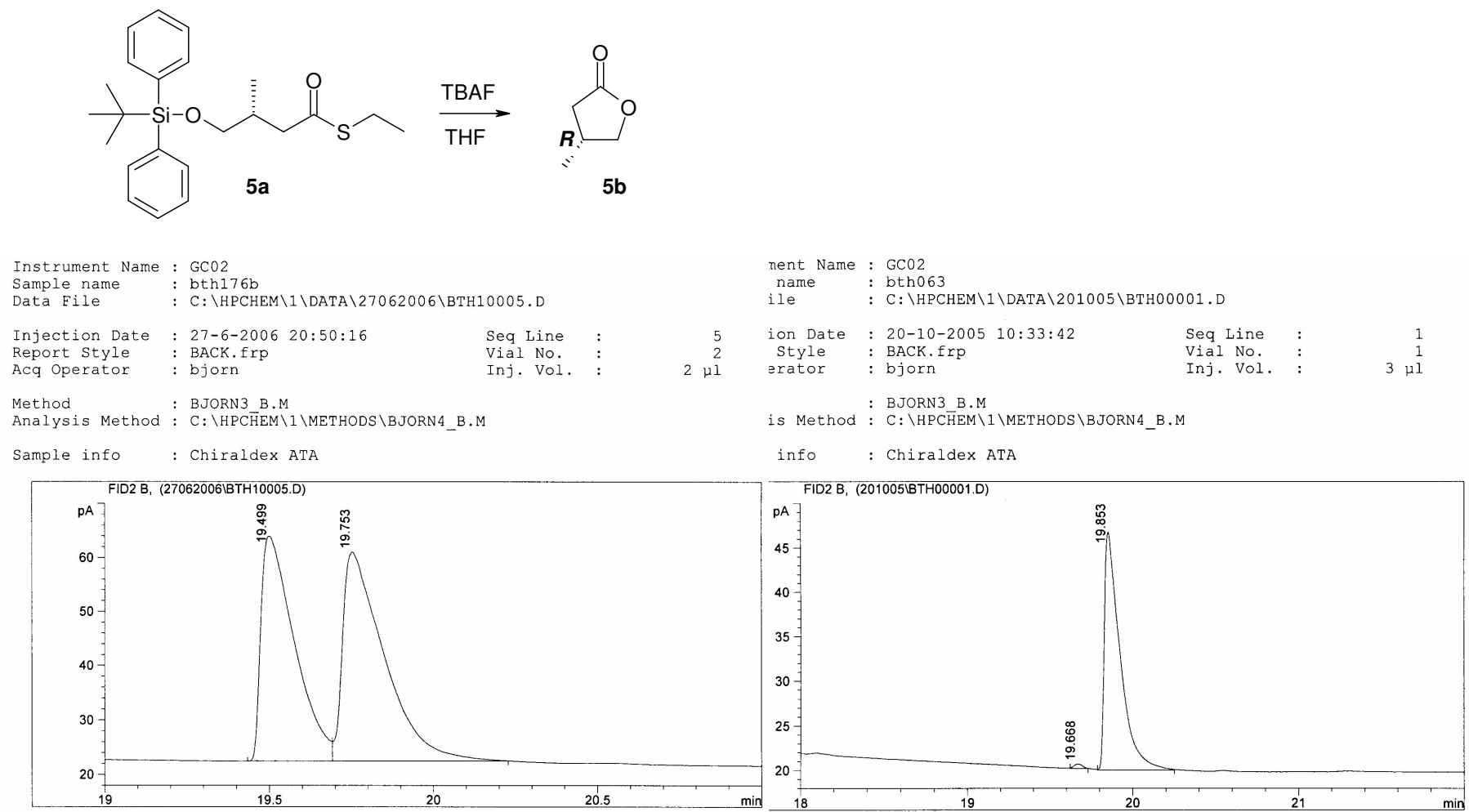

Signal 1: FID2 B,

1: FID2 B,

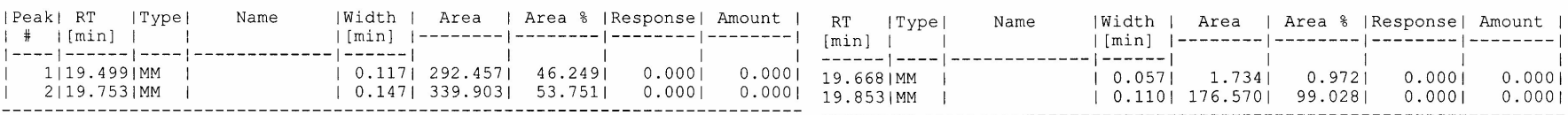

${ }^{1}$ H. G. W. Leuenberg, W. Boguth, R. Barner, M. Schmid and R. Zell, Helv. Chem. Acta., 1979, 62, 455 463.

${ }^{2}$ T. Mukayama, K. Fujimoto, T. Hirose and T. Takeda, Chem. Lett., 1980, 635

${ }^{3}$ K. Mori, Tetrahedron 1983, 39, $3107-3109$. 


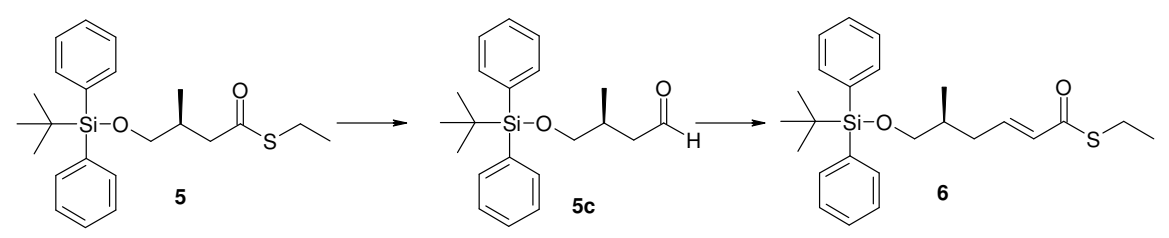

(-)-(E)-(S)-6-(tert-Butyl-diphenyl-silanyloxy)-5-methyl-hex-2-enethioic acid S-ethyl ester (6)

To a stirred mixture of 5 (2.0 g, $5 \mathrm{mmol})$ and $10 \% \mathrm{Pd}-\mathrm{C}(5 \mathrm{~mol} \%, 267 \mathrm{mg})$ in $\mathrm{CH}_{2} \mathrm{Cl}_{2}(10 \mathrm{~mL})$ was added 3 equiv. of $\mathrm{Et}_{3} \mathrm{SiH}(2.41 \mathrm{~mL}, 15.0 \mathrm{mmol})$ at $\mathrm{rt}$ under nitrogen. Stirring was continued at rt until the reduction was completed (10-30 min). The catalyst was filtered off through a Celite path and washed with $\mathrm{CH}_{2} \mathrm{Cl}_{2}$. The filtrate was concentrated under reduced pressure and purified by flash chromatography (eluent pentane/ether 40:1) to give 5c which was used in the next step without complete removal of the eluent. A solution of 5c and $\mathrm{Ph}_{3} \mathrm{PCHCOSEt}(2.366 \mathrm{~g}, 6.50 \mathrm{mmol})$ in $\mathrm{CH}_{2} \mathrm{Cl}_{2}(30 \mathrm{~mL})$ was refluxed for $24 \mathrm{~h}$. The solution was concentrated under reduced pressure and purified by flash chromatography (eluent pentane/ether 40:1) to afford $\alpha, \beta$-unsaturated thioester 6 as a colourless oil $(1.491 \mathrm{~g}, 70 \%$ yield over 2 steps, $\left.[\alpha]_{\mathrm{D}}=-5.73\left(c=1.57, \mathrm{CHCl}_{3}\right)\right) .{ }^{1} \mathrm{H}-\mathrm{NMR}\left(400 \mathrm{MHz}, \mathrm{CDCl}_{3}\right): \delta 7.65(\mathrm{~d}, J=7.9 \mathrm{~Hz}, 4 \mathrm{H}), 7.41(\mathrm{~m}, 6 \mathrm{H})$, $6.87(\mathrm{dt}, J=15.4,7.6 \mathrm{~Hz}, 1 \mathrm{H}), 6.11(\mathrm{dt}, J=15.5,1.4 \mathrm{~Hz}, 1 \mathrm{H}), 3.53(\mathrm{dd}, J=10.0,5.4 \mathrm{~Hz}, 1 \mathrm{H}), 3.46(\mathrm{dd}, J=$ 10.0, $6.4 \mathrm{~Hz}, 1 \mathrm{H}), 2.95(\mathrm{q}, J=7.4 \mathrm{~Hz}, 2 \mathrm{H}), 2.44(\mathrm{~m}, 1 \mathrm{H}), 2.05(\mathrm{~m}, 1 \mathrm{H}), 1.86(\mathrm{~m}, 1 \mathrm{H}), 1.29(\mathrm{t}, J=7.4 \mathrm{~Hz}$, 3H), $1.06(\mathrm{~s}, 9 \mathrm{H}), 0.92$ (d, $J=6.8 \mathrm{~Hz}, 3 \mathrm{H}) .{ }^{13} \mathrm{C}-\mathrm{NMR}\left(100.6 \mathrm{MHz}, \mathrm{CDCl}_{3}\right): 189.97$ (s), 143.89 (d), 135.57 (d), $133.70(\mathrm{~s}), 129.95$ (d), 129.61 (d), 127.64 (d), 68.07 (t), 35.97 (t), 35.42 (d), 26.86 (q), 23.03 (t), 19.29 (s), 16.46 (q), 14.83 (q). $\mathrm{MS}(\mathrm{EI}+)$ for $\mathrm{C}_{25} \mathrm{H}_{34} \mathrm{O}_{2} \mathrm{SSi}: \mathrm{m} / \mathrm{z}(\%)=369$ (100\%, M - t-Butyl), MS(CI+) for $\mathrm{C}_{25} \mathrm{H}_{34} \mathrm{O}_{2} \mathrm{SSi}: \mathrm{m} / \mathrm{z}(\%)=444\left(100 \%, \mathrm{M}+\mathrm{NH}_{4}^{+}\right), 427\left(1.5 \%, \mathrm{M}+\mathrm{H}^{+}\right)$. HRMS(EI+) for $\mathrm{C}_{25} \mathrm{H}_{34} \mathrm{O}_{2} \mathrm{SSi}: \mathrm{m} / \mathrm{z}(\%)$ = 369 (100\%, M - t-Butyl), Measured Mass: 369.1331 Da, Calculated Mass: 369.1345 Da. For spectral data see: Chem. Commun., 2007, 489-491.

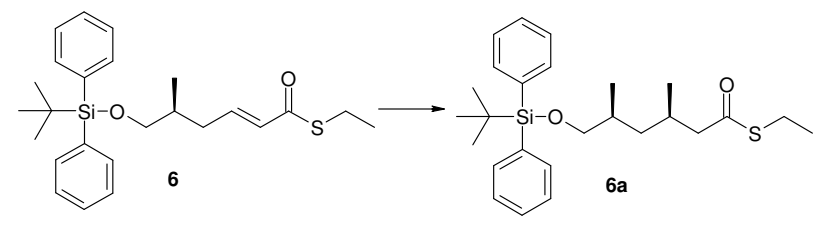

\section{(-)-(3R,5S)-6-(tert-Butyl-diphenyl-silanyloxy)-3,5-dimethyl-hexanethioic acid S-ethyl ester (6a)}

$\left(R, S_{F e}\right)$-Josiphos $(4) \cdot \mathrm{CuBr}$ complex $(16.8 \mathrm{mg}, 0.023 \mathrm{mmol}, 1 \mathrm{~mol} \%)$ was dissolved in $t$-BuOMe $(13.8 \mathrm{~mL})$ under nitrogen. The mixture was cooled to $-75^{\circ} \mathrm{C}$ and methylmagnesium bromide $(2.73$ mmol, solution in diethyl ether) was added dropwise over $10 \mathrm{~min}$. After stirring for $10 \mathrm{~min}$, a solution of thioester 6 (969 mg, $2.275 \mathrm{mmol})$ in $t$-BuOMe $(3.9 \mathrm{~mL})$ was added via syringe pump over $1 \mathrm{~h}$. The reaction mixture was stirred at $-75^{\circ} \mathrm{C}$ for $17 \mathrm{~h}$, then quenched by the addition of $\mathrm{MeOH}$ and allowed to warm to room temperature. Saturated aqueous $\mathrm{NH}_{4} \mathrm{Cl}$ solution was then added. After phase separation and extraction of the aqueous phase with 3 portions of diethyl ether, the combined organic phases were dried over $\mathrm{MgSO}_{4}$, concentrated under reduced pressure and purified by flash chromatography (eluent pentane/ether 40:1) to afford $\mathbf{6 a}$ as a colourless oil $(900 \mathrm{mg}, 90 \%$ yield, syn/ant ratio by NMR $=96 / 4$, d.r.calculated from syn/anti ratio $=$ $\left.(3 R, 5 S):(3 S, 5 S):(3 R, 5 R):(3 S, 5 R)=97: 2: 1: 0,[\alpha]_{\mathrm{D}}=-4.61\left(c=1.91, \mathrm{CHCl}_{3}\right)\right) .{ }^{1} \mathrm{H}-\mathrm{NMR}\left(400 \mathrm{MHz} \mathrm{CDCl}_{3}\right)$ : $\delta 7.67(\mathrm{dd}, J=7.7,1.6 \mathrm{~Hz}, 4 \mathrm{H}), 7.41(\mathrm{~m}, 6 \mathrm{H}), 3.50(\mathrm{dd}, J=9.9,5.5 \mathrm{~Hz}, 1 \mathrm{H}), 3.43(\mathrm{dd}, J=9.9,6.4 \mathrm{~Hz}, 1 \mathrm{H})$, $2.87(\mathrm{q}, J=7.4 \mathrm{~Hz}, 2 \mathrm{H}), 2.52(\mathrm{dd}, J=14.4,5.1 \mathrm{~Hz}, 1 \mathrm{H}), 2.25(\mathrm{dd}, J=14.4,8.8 \mathrm{~Hz}, 1 \mathrm{H}), 2.08(\mathrm{~m}, 1 \mathrm{H}), 1.71$ $(\mathrm{m}, 1 \mathrm{H}), 1.41(\mathrm{~m}, 1 \mathrm{H}), 1.24(\mathrm{t}, J=7.4 \mathrm{~Hz}, 3 \mathrm{H}), 1.06(\mathrm{~s}, 9 \mathrm{H}), 1.03(\mathrm{~m}, 1 \mathrm{H}), 0.94(\mathrm{~d}, J=6.7 \mathrm{~Hz}, 3 \mathrm{H}), 0.91(\mathrm{~d}$, $J=6.6 \mathrm{~Hz}, 3 \mathrm{H}) .{ }^{13} \mathrm{C}-\mathrm{NMR}\left(100.6 \mathrm{MHz}, \mathrm{CDCl}_{3}\right): 199.15(\mathrm{~s}), 135.61(\mathrm{~d}), 133.94$ (s), 129.50 (d), 127.57 (d), $68.74(\mathrm{t}), 51.19(\mathrm{t}), 40.79(\mathrm{t}), 33.16(\mathrm{~d}), 28.69(\mathrm{~d}), 26.88(\mathrm{q}), 23.26(\mathrm{t}), 20.28(\mathrm{q}), 19.29(\mathrm{~s}), 17.42(\mathrm{q}), 14.79$ (q). $\mathrm{MS}(\mathrm{EI}+)$ for $\mathrm{C}_{26} \mathrm{H}_{38} \mathrm{O}_{2} \mathrm{SSi}: \mathrm{m} / \mathrm{z}(\%)=385$ (100\%, $\mathrm{M}-t$-Butyl), $\mathrm{MS}(\mathrm{CI}+)$ for $\mathrm{C}_{26} \mathrm{H}_{38} \mathrm{O}_{2} \mathrm{SSi}: \mathrm{m} / \mathrm{z}(\%)=$ $460\left(100 \%, \mathrm{M}+\mathrm{NH}_{4}^{+}\right), 443\left(12.5 \%, \mathrm{M}+\mathrm{H}^{+}\right)$. HRMS(EI+) for $\mathrm{C}_{26} \mathrm{H}_{38} \mathrm{O}_{2} \mathrm{SSi}: \mathrm{m} / \mathrm{z}(\%)=385(100 \%, \mathrm{M}-t$ - 
Butyl), Measured Mass: 385.1668 Da, Calculated Mass: 385.1658 Da. For spectral data see: Chem. Commun., 2007, 489-491.

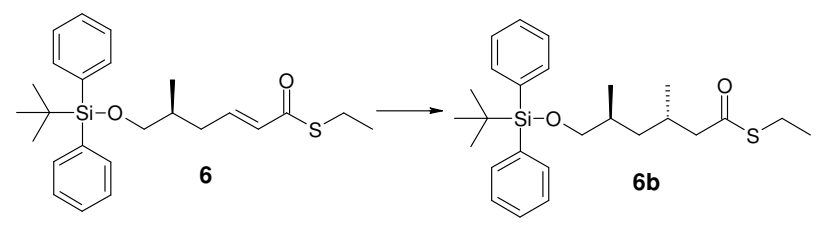

(3S,5S)-6-(tert-Butyl-diphenyl-silanyloxy)-3,5-dimethyl-hexanethioic acid S-ethyl ester (6b)

$\left(S, R_{F e}\right)$-Josiphos $(4) \cdot \mathrm{CuBr}$ complex $(3 \mathrm{mg}, 0.004 \mathrm{mmol}, 5 \mathrm{~mol} \%)$ was dissolved in $t$-BuOMe $(0.8 \mathrm{~mL})$ under nitrogen. The mixture was cooled to $-75^{\circ} \mathrm{C}$ and methylmagnesium bromide $(0.12 \mathrm{mmol}$, solution in diethyl ether) was added dropwise over $10 \mathrm{~min}$. After stirring for $10 \mathrm{~min}$, a solution of thioester 6 (40 $\mathrm{mg}$, $0.094 \mathrm{mmol})$ in $t$-BuOMe $(0.2 \mathrm{~mL})$ was added via syringe pump over $1 \mathrm{~h}$. The reaction mixture was stirred at $-75^{\circ} \mathrm{C}$ for $17 \mathrm{~h}$, then quenched by the addition of $\mathrm{MeOH}$ and allowed to warm to room temperature. Saturated aqueous $\mathrm{NH}_{4} \mathrm{Cl}$ solution was then added. After phase separation and extraction of the aqueous phase with 3 portions of diethyl ether, the combined organic phases were dried over $\mathrm{MgSO}_{4}$, concentrated under reduced pressure and purified by flash chromatography (eluent pentane/ether 40:1) to afford $\mathbf{6 b}$ as a colourless oil (38 mg, 90\% yield, syn/anti by NMR > 5/95, ${ }^{1} \mathrm{H}-\mathrm{NMR}\left(400 \mathrm{MHz}, \mathrm{CDCl}_{3}\right): \delta 7.67(\mathrm{dd}, J=$ 7.7, $1.6 \mathrm{~Hz}, 4 \mathrm{H}), 7.41(\mathrm{~m}, 6 \mathrm{H}), 3.47(\mathrm{~m}, 2 \mathrm{H}), 2.87(\mathrm{q}, J=7.5 \mathrm{~Hz}, 2 \mathrm{H}), 2.47(\mathrm{dd}, J=14.4,6.3 \mathrm{~Hz}, 1 \mathrm{H}), 2.37$ $(\mathrm{dd}, J=14.5,7.7 \mathrm{~Hz}, 1 \mathrm{H}), 2.10(\mathrm{~m}, 1 \mathrm{H}), 1.73(\mathrm{~m}, 1 \mathrm{H}), 1.28(\mathrm{~m}, 1 \mathrm{H}), 1.25(\mathrm{t}, J=7.4 \mathrm{~Hz}, 3 \mathrm{H}), 1.08(\mathrm{~m}, 1 \mathrm{H})$, $1.06(\mathrm{~s}, 9 \mathrm{H}), 0.90(\mathrm{~d}, J=6.6 \mathrm{~Hz}, 6 \mathrm{H}) .{ }^{13} \mathrm{C}-\mathrm{NMR}\left(100.6 \mathrm{MHz}, \mathrm{CDCl}_{3}\right): 199.10$ (s), 135.61 (d), 134.00 (s), 129.49 (d), 127.56 (d), 69.29 (t), 52.14 (t), 40.13 (t), 33.11 (d), 28.4 (d), 26.88 (q), 23.25 (t), 19.30 (s), 19.17 (q), 16.36 (q), 14.81 (q). For spectral data see: Chem. Commun., 2007, 489-491.

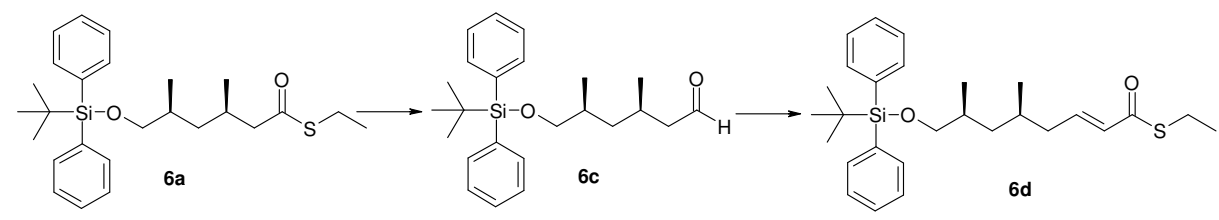

(-)-(E)-(5S,7S)-8-(tert-Butyl-diphenyl-silanyloxy)-5,7-dimethyl-oct-2-enethioic acid S-ethyl ester (6d)

To a stirred mixture of $6 \mathbf{a}(290 \mathrm{mg}, 0.656 \mathrm{mmol})$ and $10 \% \mathrm{Pd}-\mathrm{C}(5 \mathrm{~mol} \%, 35 \mathrm{mg})$ in $\mathrm{CH}_{2} \mathrm{Cl}_{2}(1.33 \mathrm{~mL})$ was added 3 equiv of $\mathrm{Et}_{3} \mathrm{SiH}(0.316 \mathrm{~mL}, 1.968 \mathrm{mmol})$ at $\mathrm{rt}$ under nitrogen. Stirring was continued at rt until the reduction was completed (10-30 min). The catalyst was filtered off through a Celite path and washed with the solvent of the reaction. The filtrate was concentrated under reduced pressure and purified by flash chromatography (eluent pentane/ether 40:1) to give aldehyde $\mathbf{6 c}$ which was used in the next step without complete removal of the eluent. A solution of aldehyde $\mathbf{6 c}$ and $\mathrm{Ph}_{3} \mathrm{PCHCOSEt}(287 \mathrm{mg}, 0.787 \mathrm{mmol})$ in $\mathrm{CH}_{2} \mathrm{Cl}_{2}(6.7 \mathrm{~mL})$ was refluxed for $24 \mathrm{~h}$. The solution was concentrated under reduced pressure and purified by flash chromatography (eluent pentane/ether $40: 1$ ) to afford $\alpha, \beta$-unsaturated thioester $\mathbf{6 d}$ as a colourless oil $\left(214 \mathrm{mg}, 70 \%\right.$ yield over 2 steps, $\left.[\alpha]_{\mathrm{D}}=-7.6\left(c=1.97, \mathrm{CHCl}_{3}\right)\right)$. ${ }^{1} \mathrm{H}-\mathrm{NMR}\left(400 \mathrm{MHz}, \mathrm{CDCl}_{3}\right): \delta 7.66$ $(\mathrm{dd}, J=7.7,1.6 \mathrm{~Hz}, 4 \mathrm{H}), 7.41(\mathrm{~m}, 6 \mathrm{H}), 6.83(\mathrm{dt}, J=15.4,7.6 \mathrm{~Hz}, 1 \mathrm{H}), 6.08(\mathrm{dt}, J=15.5,1.4 \mathrm{~Hz}, 1 \mathrm{H})$, $3.50(\mathrm{dd}, J=9.8,5.3 \mathrm{~Hz}, 1 \mathrm{H}), 3.42(\mathrm{dd}, J=9.8,6.3 \mathrm{~Hz}, 1 \mathrm{H}), 2.94(\mathrm{q}, J=7.4 \mathrm{~Hz}, 2 \mathrm{H}), 2.18(\mathrm{~m}, 1 \mathrm{H}), 1.92$ $(\mathrm{m}, 1 \mathrm{H}), 1.69(\mathrm{~m}, 2 \mathrm{H}), 1.39(\mathrm{~m}, 1 \mathrm{H}), 1.28(\mathrm{t}, J=7.4 \mathrm{~Hz}, 3 \mathrm{H}), 1.06(\mathrm{~s}, 9 \mathrm{H}), 1.02(\mathrm{~m}, 1 \mathrm{H}), 0.93(\mathrm{~d}, J=6.7 \mathrm{~Hz}$, 3H), 0.85 (d, $J=6.6 \mathrm{~Hz}, 3 \mathrm{H}) .{ }^{13} \mathrm{C}-\mathrm{NMR}\left(100.6 \mathrm{MHz}, \mathrm{CDCl}_{3}\right.$ ): 189.97 (s), 144.07 (d), 135.59 (d), 133.93 (s), 129.84 (d), 129.51 (d), 127.57 (d), $68.61(\mathrm{t}), 40.76(\mathrm{t}), 39.45$ (t), 33.08 (d), 29.96 (d), 26.86 (q), 23.01 (t), 20.12 (q), 19.28 (s), 17.59 (q), 14.81 (q). $\mathrm{MS}(\mathrm{EI}+)$ for $\mathrm{C}_{28} \mathrm{H}_{40} \mathrm{O}_{2} \mathrm{SSi}: \mathrm{m} / \mathrm{z}(\%)=411$ (100\%, M - $t$-Butyl), $\mathrm{MS}(\mathrm{CI}+)$ for $\mathrm{C}_{28} \mathrm{H}_{40} \mathrm{O}_{2} \mathrm{SSi}: \mathrm{m} / \mathrm{z}(\%)=486\left(100 \%, \mathrm{M}+\mathrm{NH}_{4}{ }^{+}\right)$. $\mathrm{HRMS}(\mathrm{EI}+)$ for $\mathrm{C}_{28} \mathrm{H}_{40} \mathrm{O}_{2} \mathrm{SSi}: \mathrm{m} / \mathrm{z}(\%)=411$ (100\%, M - t-Butyl), Measured Mass: 411.1812 Da, Calculated Mass: 411.1814 Da. For spectral data see: Chem. Commun., 2007, 489-491. 


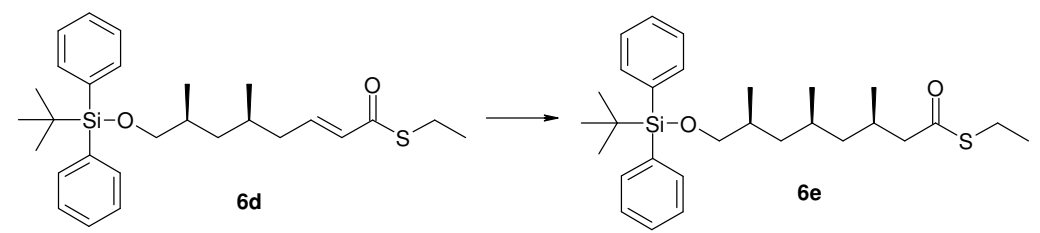

(-)-(3R,5S,7S)8-(tert-Butyl-diphenyl-silanyloxy)-3,5,7-trimethyl-octanethioic acid S-ethyl ester (6e)

$\left(R, S_{F e}\right)$-Josiphos (4)•CuBr complex $(3.2 \mathrm{mg}, 0.004 \mathrm{mmol}, 1 \mathrm{~mol} \%)$ was dissolved in $t$-BuOMe $(0.8 \mathrm{~mL})$ under nitrogen. The mixture was cooled to $-75^{\circ} \mathrm{C}$ and methylmagnesium bromide $(0.541 \mathrm{mmol}$, solution in diethyl ether) was added dropwise over $10 \mathrm{~min}$. After stirring for $10 \mathrm{~min}$, a solution of thioester $\mathbf{6 d}(211 \mathrm{mg}$, $0.451 \mathrm{mmol})$ in $t$-BuOMe $(2.76 \mathrm{~mL})$ was added via syringe pump over $1 \mathrm{~h}$. The reaction mixture was stirred at $-75{ }^{\circ} \mathrm{C}$ for $17 \mathrm{~h}$, then quenched by the addition of $\mathrm{MeOH}$ and allowed to warm to room temperature. Saturated aqueous $\mathrm{NH}_{4} \mathrm{Cl}$ solution was then added. After phase separation and extraction of the aqueous phase with 3 portions of diethyl ether, the combined organic phases were dried over $\mathrm{MgSO}_{4}$, concentrated under reduced pressure and purified by flash chromatography (eluent pentane/ether 40:1) to afford $6 \mathbf{e}$ as a colourless oil $(185 \mathrm{mg}, 85 \%$ yield, syn/anti by NMR $=>96 / 4$, d.r.calculated from syn/anti ratio $=(3 R, 5 S, 7 S):(3 S, 5 S, 7 S):(3 R, 5 R, 7 S):(3 R, 5 S, 7 R)=95: 2: 2: 1$, diastereoisomers less than 0.04 were neglected, $\left.[\alpha]_{\mathrm{D}}=-6.8\left(c=1.13, \mathrm{CHCl}_{3}\right)\right) .{ }^{1} \mathrm{H}-\mathrm{NMR}\left(400 \mathrm{MHz}, \mathrm{CDCl}_{3}\right): \delta 7.67(\mathrm{dd}, J=1.7,7.7 \mathrm{~Hz}, 4 \mathrm{H})$, $7.41(\mathrm{~m}, 6 \mathrm{H}), 3.46(\mathrm{dd}, J=9.8,5.1 \mathrm{~Hz}, 1 \mathrm{H}), 3.41,(\mathrm{dd}, J=9.8,6.5 \mathrm{~Hz}, 1 \mathrm{H}), 2.87(\mathrm{q}, J=7.4 \mathrm{~Hz}, 2 \mathrm{H}), 2.52$ $(\mathrm{dd}, J=5.0,14.3 \mathrm{~Hz}, 1 \mathrm{H}), 2.23(\mathrm{dd}, J=8.8,14.3 \mathrm{~Hz}, 1 \mathrm{H}), 2.10(\mathrm{~m}, 1 \mathrm{H}), 1.72(\mathrm{~m}, 1 \mathrm{H}), 1.49(\mathrm{~m}, 1 \mathrm{H}), 1.35$ $(\mathrm{m}, 1 \mathrm{H}), 1.25(\mathrm{t}, J=7.4 \mathrm{~Hz}, 3 \mathrm{H}), 1.21(\mathrm{~m}, 1 \mathrm{H}), 1.06(\mathrm{~s}, 12 \mathrm{H}), 0.94(\mathrm{~d}, J=6.7 \mathrm{~Hz}, 3 \mathrm{H}), 0.92(\mathrm{~m}, 2 \mathrm{H}), 0.91$ $(\mathrm{d}, J=6.5 \mathrm{~Hz}, 3 \mathrm{H}), 0.84(\mathrm{~d}, J=6.5 \mathrm{~Hz}, 3 \mathrm{H}) .{ }^{13} \mathrm{C}-\mathrm{NMR}\left(100.6 \mathrm{MHz}, \mathrm{CDCl}_{3}\right): 199.22(\mathrm{~s}), 135.60$ (d), 134.03 $(\mathrm{s}), 129.47(\mathrm{~d}), 127.54(\mathrm{~d}), 68.74(\mathrm{t}), 50.93(\mathrm{t}), 44.71(\mathrm{t}), 41.18(\mathrm{t}), 33.08(\mathrm{~d}), 28.59(\mathrm{~d}), 27.61(\mathrm{~d}), 26.88(\mathrm{q})$, 23.24 (t), 20.53 (q), 20.46 (q), 19.29 (s), 17.98 (q), 14.80 (q). MS(EI+) for $\mathrm{C}_{29} \mathrm{H}_{44} \mathrm{O}_{2} \mathrm{SSi}: \mathrm{m} / \mathrm{z}(\%)=427$ (100\%, M - t-Butyl), MS(CI+) for $\mathrm{C}_{29} \mathrm{H}_{44} \mathrm{O}_{2} \mathrm{SSi}: \mathrm{m} / \mathrm{z}(\%)=502\left(100 \%, \mathrm{M}+\mathrm{NH}_{4}^{+}\right)$. HRMS(EI+) for $\mathrm{C}_{29} \mathrm{H}_{44} \mathrm{O}_{2} \mathrm{SSi}: \mathrm{m} / \mathrm{z}(\%)=427$ (100\%, M - t-Butyl), Measured Mass: 427.2142 Da, Calculated Mass: 427.2127 Da. For spectral data see: Chem. Commun., 2007, 489-491.

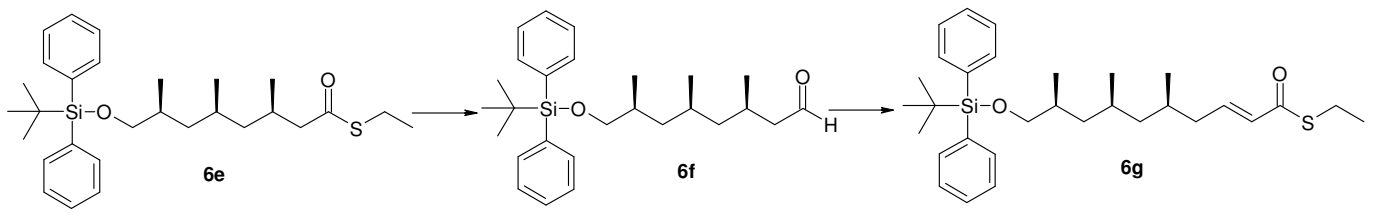

(-)-(E)-(5R,7S,9S)-10-(tert-Butyl-diphenyl-silanyloxy)-5,7,9-trimethyl-dec-2-enethioic acid S-ethyl ester (6g)

To a stirred mixture of $6 \mathrm{e}(1.500 \mathrm{~g}, 3.099 \mathrm{mmol})$ and $10 \% \mathrm{Pd}-\mathrm{C}(5 \mathrm{~mol} \%, 165 \mathrm{mg})$ in $\mathrm{CH}_{2} \mathrm{Cl}_{2}(6.4 \mathrm{~mL})$ was added 3 equiv of $\mathrm{Et}_{3} \mathrm{SiH}(1.494 \mathrm{~mL}, 9.297 \mathrm{mmol})$ at $\mathrm{rt}$ under nitrogen. Stirring was continued at $\mathrm{rt}$ until the reduction was completed (10-30 min). The catalyst was filtered off through a Celite path and washed with the solvent of the reaction. The filtrate was concentrated under reduced pressure and purified by flash chromatography (eluent pentane/ether 40:1) to give aldehyde 6 f which was used in the next step without complete removal of the eluent. A solution of aldehyde $\mathbf{6 f}$ and $\mathrm{Ph}_{3}$ PCHCOSEt (1.466 $\left.\mathrm{g}, 4.029 \mathrm{mmol}\right)$ in $\mathrm{CH}_{2} \mathrm{Cl}_{2}(32 \mathrm{~mL})$ was refluxed for $24 \mathrm{~h}$. The solution was concentrated under reduced pressure and purified by flash chromatography (eluent pentane/ether $40: 1$ ) to afford $\alpha, \beta$-unsaturated thioester $\mathbf{6 g}$ as a colourless oil $\left(1.102 \mathrm{~g}, 70 \%\right.$ yield over 2 steps, $\left.[\alpha]_{\mathrm{D}}=-8.2\left(c=0.828, \mathrm{CHCl}_{3}\right)\right)$. ${ }^{1} \mathrm{H}-\mathrm{NMR}\left(400 \mathrm{MHz}, \mathrm{CDCl}_{3}\right): \delta 7.67$ (dd, $J=7.9,1.6 \mathrm{~Hz}, 4 \mathrm{H}), 7.40(\mathrm{~m}, 6 \mathrm{H}), 6.86(\mathrm{dt}, J=15.5,8.0 \mathrm{~Hz}, 1 \mathrm{H}), 6.09$ (dt, $J=15.5,1.4 \mathrm{~Hz}, 1 \mathrm{H}), 3.51$ $(\mathrm{dd}, J=9.8,5.2 \mathrm{~Hz}, 1 \mathrm{H}), 3.42(\mathrm{dd}, J=9.8,6.4 \mathrm{~Hz}, 1 \mathrm{H}), 2.95(\mathrm{q}, J=7.4 \mathrm{~Hz}, 2 \mathrm{H}), 2.21(\mathrm{~m}, 1 \mathrm{H}), 1.90(\mathrm{~m}$, $1 \mathrm{H}), 1.72(\mathrm{~m}, 2 \mathrm{H}), 1.52(\mathrm{~m}, 1 \mathrm{H}), 1.36(\mathrm{~m}, 1 \mathrm{H}), 1.29(\mathrm{t}, J=7.4 \mathrm{~Hz}, 3 \mathrm{H}), 1.21(\mathrm{~m}, 1 \mathrm{H}), 1.07(\mathrm{~s}, 9 \mathrm{H}), 0.94(\mathrm{~d}, J$ $=6.7 \mathrm{~Hz}, 3 \mathrm{H}), 0.91(\mathrm{~m}, 2 \mathrm{H}), 0.86(\mathrm{~d}, J=6.6 \mathrm{~Hz}, 3 \mathrm{H}), 0.83(\mathrm{~d}, J=6.5 \mathrm{~Hz}, 3 \mathrm{H}) .{ }^{13} \mathrm{C}-\mathrm{NMR}(100.6 \mathrm{MHz}$, $\mathrm{CDCl}_{3}$ ): 189.96 (s), 144.17 (d), 135.59 (d), 134.03 (s), 129.83 (d), 129.47 (d), 127.53 (d), 68.76 (t), 44.78 $(\mathrm{t}), 41.24(\mathrm{t}), 39.10(\mathrm{t}), 33.14(\mathrm{~d}), 29.90(\mathrm{~d}), 27.61(\mathrm{~d}), 26.88(\mathrm{q}), 23.01(\mathrm{t}), 20.70(\mathrm{q}), 20.42(\mathrm{q}), 19.30(\mathrm{~s})$, 17.97 (q), 14.81 (q). $\mathrm{MS}(\mathrm{EI}+)$ for $\mathrm{C}_{31} \mathrm{H}_{46} \mathrm{O}_{2} \mathrm{SSi}: \mathrm{m} / \mathrm{z}(\%)=453(100 \%, \mathrm{M}-t$-Butyl), MS(CI+) for 
$\mathrm{C}_{31} \mathrm{H}_{46} \mathrm{O}_{2} \mathrm{SSi}: \mathrm{m} / \mathrm{z}(\%)=528\left(100 \%, \mathrm{M}+\mathrm{NH}_{4}^{+}\right)$. HRMS(EI+) for $\mathrm{C}_{31} \mathrm{H}_{46} \mathrm{O}_{2} \mathrm{SSi}: \mathrm{m} / \mathrm{z}(\%)=453(100 \%, \mathrm{M}-$ $t$-Butyl), Measured Mass: 453.2264 Da, Calculated Mass: 453.2284 Da. For spectral data see: Chem. Commun., 2007, 489-491.

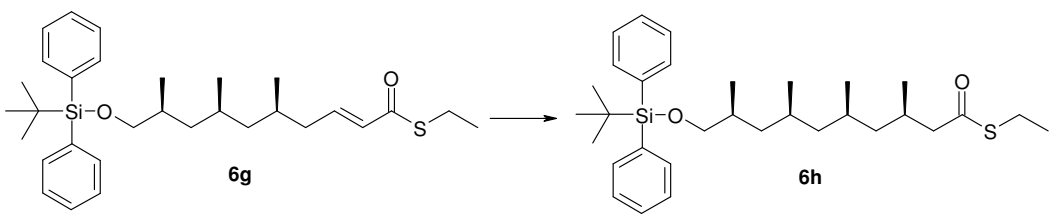

(-)-(3R,5R,7S,9S)-10-(tert-Butyl-diphenyl-silanyloxy)-3,5,7,9-tetramethyl-decanethioic acid S-ethyl ester (6h)

$\left(R, S_{F e}\right)$-Josiphos (4) $\bullet \mathrm{CuBr}$ complex $(15.9 \mathrm{mg}, 0.022 \mathrm{mmol}, 1 \mathrm{~mol} \%)$ was dissolved in $t$-BuOMe $(13.6 \mathrm{~mL})$ under nitrogen. The mixture was cooled to $-75^{\circ} \mathrm{C}$ and methylmagnesium bromide $(2.593 \mathrm{mmol}$, solution in diethyl ether) was added dropwise. After stirring for $10 \mathrm{~min}$, a solution of thioester $\mathbf{6 g}(1.102 \mathrm{~g}, 2.161$ $\mathrm{mmol})$ in $t$-BuOMe $(3.9 \mathrm{~mL})$ was added via syringe pump over $1 \mathrm{~h}$. The reaction mixture was stirred at -75 ${ }^{\circ} \mathrm{C}$ for $17 \mathrm{~h}$, then quenched by the addition of $\mathrm{MeOH}$ and allowed to warm to room temperature. Saturated aqueous $\mathrm{NH}_{4} \mathrm{Cl}$ solution was then added. After phase separation and extraction of the aqueous phase with 3 portions of diethyl ether, the combined organic phases were dried over $\mathrm{MgSO}_{4}$, concentrated under reduced pressure and purified by flash chromatography (eluent pentane/ether 40:1) to afford $\mathbf{6 h}$ as a colourless oil $(1.010 \mathrm{mg}, 88 \%$ yield, syn/ant ratio by $\mathrm{NMR}=>96 / 4$, d.r. calculated from syn/anti ratio $=$ $(3 R, 5 R, 7 S, 9 S):(3 S, 5 R, 7 S, 9 S):(3 R, 5 S, 7 S, 9 S):(3 R, 5 R, 7 R, 9 S):(3 R, 5 R, 7 S, 9 R)=94: 1: 2: 2: 1$, diastereoisomers less than 0.04 were neglected $\left.[\alpha]_{\mathrm{D}}=-6.7\left(c=1.14, \mathrm{CHCl}_{3}\right)\right)$. ${ }^{1} \mathrm{H}-\mathrm{NMR}\left(400 \mathrm{MHz}, \mathrm{CDCl}_{3}\right): \delta 7.68(\mathrm{dd}, J=$ $7.8,1.6 \mathrm{~Hz}, 4 \mathrm{H}), 7.41(\mathrm{~m}, 6 \mathrm{H}), 3.53(\mathrm{dd}, J=9.8,5.0 \mathrm{~Hz}, 1 \mathrm{H}), 3.43(\mathrm{dd}, J=9.8,6.4 \mathrm{~Hz}, 1 \mathrm{H}), 2.88(\mathrm{q}, J=$ $7.4 \mathrm{~Hz}, 2 \mathrm{H}), 2.56(\mathrm{dd}, J=14.3 \mathrm{~Hz}, J=5.0 \mathrm{~Hz}, 1 \mathrm{H}), 2.26(\mathrm{dd}, J=14.3,8.8 \mathrm{~Hz}, 1 \mathrm{H}), 2.12(\mathrm{~m}, 1 \mathrm{H}), 1.74(\mathrm{~m}$, $1 \mathrm{H}), 1.54(\mathrm{~m}, 2 \mathrm{H}), 1.38(\mathrm{~m}, 1 \mathrm{H}), 1.26(\mathrm{t}, J=7.4 \mathrm{~Hz}, 3 \mathrm{H}), 1.20(\mathrm{~m}, 2 \mathrm{H}), 1.17(\mathrm{~s}, 9 \mathrm{H}), 0.95(\mathrm{~d}, J=6.6 \mathrm{~Hz}$, $3 \mathrm{H}), 0.94(\mathrm{~d}, J=6.5 \mathrm{~Hz}, 3 \mathrm{H}), 0.89(\mathrm{~m}, 3 \mathrm{H}), 0.85(\mathrm{~d}, J=6.5 \mathrm{~Hz}, 3 \mathrm{H}), 0.82(\mathrm{~d}, J=6.5 \mathrm{~Hz}, 3 \mathrm{H}) .{ }^{13} \mathrm{C}-\mathrm{NMR}$ $\left(100.6 \mathrm{MHz}, \mathrm{CDCl}_{3}\right): 199.24$ (s), 135.61 (d), 134.04 (s), 129.44 (d), 127.52 (d), 68.69 (t), 50.93 (t), 45.48 $(\mathrm{t}), 44.46(\mathrm{t}), 41.15(\mathrm{t}), 33.16(\mathrm{~d}), 28.63(\mathrm{~d}), 27.55(\mathrm{~d}), 27.43(\mathrm{~d}), 26.88(\mathrm{q}), 23.25(\mathrm{t}), 20.92(\mathrm{q}), 20.75(\mathrm{q})$, 20.55 (q), 19.30 (s), 18.18 (q), 14.81 (q). $\mathrm{MS}(\mathrm{EI}+)$ for $\mathrm{C}_{32} \mathrm{H}_{50} \mathrm{O}_{2} \mathrm{SSi}: \mathrm{m} / \mathrm{z}(\%)=469$ (100\%, M - $t$-Butyl), $\mathrm{MS}(\mathrm{CI}+)$ for $\mathrm{C}_{32} \mathrm{H}_{50} \mathrm{O}_{2} \mathrm{SSi}: \mathrm{m} / \mathrm{z}(\%)=544\left(100 \%, \mathrm{M}+\mathrm{NH}_{4}{ }^{+}\right)$. $\mathrm{HRMS}(\mathrm{EI}+)$ for $\mathrm{C}_{32} \mathrm{H}_{50} \mathrm{O}_{2} \mathrm{SSi}: \mathrm{m} / \mathrm{z}(\%)=469$ (100\%, M - t-Butyl), Measured Mass: 469.2590 Da, Calculated Mass: 469.2597 Da. For spectral data see: Chem. Commun., 2007, 489-491.

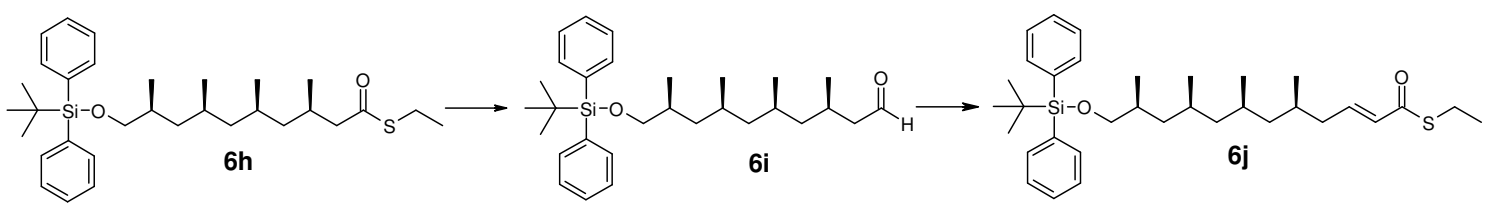
(-)-(5R,7R,9S,11S)-12-(tert-Butyl-diphenyl-silanyloxy)-5,7,9,11-tetramethyl-dodec-2-enethioic acid Sethyl ester (6j)

To a stirred mixture of $\mathbf{6 h}(1.000 \mathrm{~g}, 1.901 \mathrm{mmol})$ and $10 \% \mathrm{Pd}-\mathrm{C}(5 \mathrm{~mol} \%, 101 \mathrm{mg})$ in $\mathrm{CH}_{2} \mathrm{Cl}_{2}(3.9 \mathrm{~mL})$ was added 3 equiv of $\mathrm{Et}_{3} \mathrm{SiH}(0.916 \mathrm{~mL}, 5.703 \mathrm{mmol})$ at $\mathrm{rt}$ under nitrogen. Stirring was continued at rt until the reduction was completed $(10-30 \mathrm{~min})$. The catalyst was filtered off through a Celite path and washed with the solvent of the reaction. The filtrate was concentrated under reduced pressure and purified by flash chromatography (eluent pentane/ether 40:1) to give aldehyde 6i which was used in the next step without complete removal of the eluent. A solution of aldehyde $\mathbf{6 i}$ and $\mathrm{Ph}_{3}$ PCHCOSEt $(0.920 \mathrm{~g}, 2.471 \mathrm{mmol})$ in $\mathrm{CH}_{2} \mathrm{Cl}_{2}(20 \mathrm{~mL})$ was refluxed for $24 \mathrm{~h}$. The solution was concentrated under reduced pressure and purified by flash chromatography (eluent pentane/ether $40: 1$ ) to afford $\alpha, \beta$-unsaturated thioester $\mathbf{6 j}$ as a colourless oil $\left(735 \mathrm{mg}, 70 \%\right.$ yield over 2 steps, $\left.[\alpha]_{\mathrm{D}}=-7.24\left(c=1.08, \mathrm{CHCl}_{3}\right)\right)$. ${ }^{1} \mathrm{H}-\mathrm{NMR}\left(400 \mathrm{MHz}, \mathrm{CDCl}_{3}\right): \delta 7.67$ $(\mathrm{dd}, J=7.9,1.6 \mathrm{~Hz}, 4 \mathrm{H}), 7.40(\mathrm{~m}, 6 \mathrm{H}), 6.87(\mathrm{dt}, J=15.3,7.8 \mathrm{~Hz}, 1 \mathrm{H}), 6.10(\mathrm{dt}, J=15.4,1.4 \mathrm{~Hz}, 1 \mathrm{H}), 3.52$ (dd, $J=9.8,5.0 \mathrm{~Hz}, 1 \mathrm{H}), 3.43(\mathrm{dd}, J=9.8,6.3 \mathrm{~Hz}, 1 \mathrm{H}), 2.95(\mathrm{q}, J=7.4 \mathrm{~Hz}, 2 \mathrm{H}), 2.23(\mathrm{~m}, 1 \mathrm{H}), 1.92(\mathrm{~m}$, $1 \mathrm{H}), 1.73(\mathrm{~m}, 2 \mathrm{H}), 1.54(\mathrm{~m}, 2 \mathrm{H}), 1.38(\mathrm{~m}, 1 \mathrm{H}), 1.28(\mathrm{t}, J=7.4 \mathrm{~Hz}, 3 \mathrm{H}), 1.18(\mathrm{~m}, 2 \mathrm{H}), 1.06(\mathrm{~s}, 9 \mathrm{H}), 0.94(\mathrm{~d}$, 
$J=6.7 \mathrm{~Hz}, 3 \mathrm{H}), 0.93-0.85(\mathrm{~m}, 3 \mathrm{H}), 0.89(\mathrm{~d}, J=6.7 \mathrm{~Hz}, 3 \mathrm{H}), 0.83(\mathrm{~d}, J=6.5 \mathrm{~Hz}, 3 \mathrm{H}), 0.82(\mathrm{~d}, J=6.5 \mathrm{~Hz}$, 3H). ${ }^{13} \mathrm{C}$-NMR (100.6 MHz, $\left.\mathrm{CDCl}_{3}\right): 189.96$ (s), 144.19 (d), 135.60 (d), 134.05 (s), 129.82 (d), 129.44 (d), $127.51(\mathrm{~d}), 68.64(\mathrm{t}), 45.52(\mathrm{t}), 44.42(\mathrm{t}), 41.09(\mathrm{t}), 38.97(\mathrm{t}), 33.13(\mathrm{~d}), 29.89(\mathrm{~d}), 27.53(\mathrm{~d}), 27.38(\mathrm{~d})$, 26.87 (d), 23.00 (t), 20.97 (q), 20.81 (q), 20.52 (q), 19.29 (q) (s), 18.16 (q), 14.82 (q). MS(EI+) for $\mathrm{C}_{34} \mathrm{H}_{52} \mathrm{O}_{2} \mathrm{SSi}: \mathrm{m} / \mathrm{z}(\%)=495\left(100 \%, \mathrm{M}-t\right.$-Butyl), MS(CI+) for $\mathrm{C}_{34} \mathrm{H}_{52} \mathrm{O}_{2} \mathrm{SSi}: \mathrm{m} / \mathrm{z}(\%)=570(100 \%, \mathrm{M}+$ $\left.\mathrm{NH}_{4}{ }^{+}\right)$. HRMS(EI+) for $\mathrm{C}_{34} \mathrm{H}_{52} \mathrm{O}_{2} \mathrm{SSi}: \mathrm{m} / \mathrm{z}(\%)=495(100 \%, \mathrm{M}-t$-Butyl), Measured Mass: $495.2728 \mathrm{Da}$, Calculated Mass: 495.2753 Da.

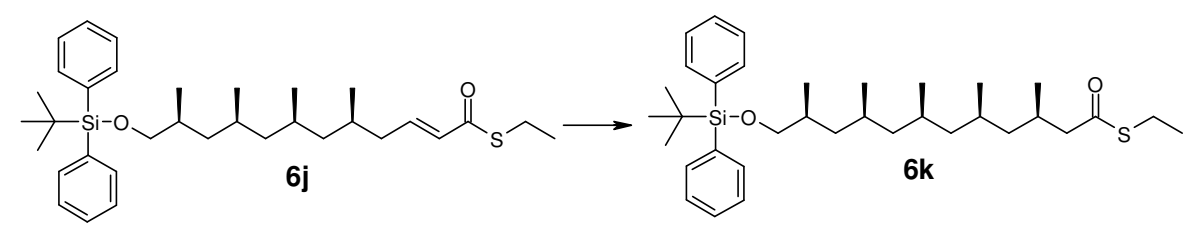

(-)-(3R,5R,7S,9S,11S)-12-(tert-Butyl-diphenyl-silanyloxy)-3,5,7,9,11-pentamethyl-dodecanethioic acid S-ethyl ester (6k)

$\left(R, S_{F e}\right)$-Josiphos $(4) \cdot \mathrm{CuBr}$ complex $(8.25 \mathrm{mg}, 0.011 \mathrm{mmol}, 1 \mathrm{~mol} \%)$ was dissolved in $t$-BuOMe $(7.037 \mathrm{~mL})$ under nitrogen. The mixture was cooled to $-75^{\circ} \mathrm{C}$ and methylmagnesium bromide $(1.344 \mathrm{mmol}$, solution in diethyl ether) was added dropwise. After stirring for $10 \mathrm{~min}$, a solution of thioester $\mathbf{6 j}$ (618 $\mathrm{mg}, 1.120 \mathrm{mmol}$ ) in $t$-BuOMe $(2.012 \mathrm{~mL})$ was added via syringe pump over $1 \mathrm{~h}$. The reaction mixture was stirred at $-75{ }^{\circ} \mathrm{C}$ for $17 \mathrm{~h}$, then quenched by the addition of $\mathrm{MeOH}$ and allowed to warm to room temperature. Saturated aqueous $\mathrm{NH}_{4} \mathrm{Cl}$ solution was then added. After phase separation and extraction of the aqueous phase with 3 portions of diethyl ether, the combined organic phases were dried over $\mathrm{MgSO}_{4}$, concentrated under reduced pressure and purified by flash chromatography (eluent pentane/ether 40:1) to afford $\mathbf{6 k}$ as a colourless oil $(559 \mathrm{mg}, 90 \%$ yield, syn/ant ratio by $\mathrm{NMR}=>96 / 4$, d.r. calculated from syn/anti ratio = $(3 R, 5 R, 7 S, 9 S, 11 S):(3 S, 5 R, 7 S, 9 S, 11 S):(3 R, 5 S, 7 S, 9 S, 11 S):(3 R, 5 S, 7 R, 9 S, 11 S):(3 R, 5 R, 7 S, 9 R, 11 S):(3 R, 5 R, 7 S, 9$ $S, 11 R)=93: 1: 1: 2: 2: 1$, diastereoisomers less than 0.04 were neglected. $\left.[\alpha]_{\mathrm{D}}=-5.3\left(c=1.10, \mathrm{CHCl}_{3}\right)\right) .{ }^{1} \mathrm{H}-$ NMR $\left(400 \mathrm{MHz}, \mathrm{CDCl}_{3}\right): \delta 7.68(\mathrm{dd}, J=7.8,1.6 \mathrm{~Hz}, 4 \mathrm{H}), 7.41(\mathrm{~m}, 6 \mathrm{H}), 3.52(\mathrm{dd}, J=9.7,5.0 \mathrm{~Hz}, 1 \mathrm{H})$, $3.42(\mathrm{dd}, J=9.6,6.4 \mathrm{~Hz}, 1 \mathrm{H}), 2.88(\mathrm{q}, J=7.4 \mathrm{~Hz}, 2 \mathrm{H}), 2.56(\mathrm{dd}, J=14.3,5.0 \mathrm{~Hz}, 1 \mathrm{H}), 2.26(\mathrm{dd}, J=14.2$, $8.7 \mathrm{~Hz}, 1 \mathrm{H}), 2.12(\mathrm{~m}, 1 \mathrm{H}), 1.74(\mathrm{~m}, 1 \mathrm{H}), 1.53(\mathrm{~m}, 3 \mathrm{H}), 1.39(\mathrm{~m}, 1 \mathrm{H}), 1.25(\mathrm{t}, J=7.4 \mathrm{~Hz}, 3 \mathrm{H}), 1.18(\mathrm{~m}, 3 \mathrm{H})$, $1.06(\mathrm{~s}, 9 \mathrm{H}), 0.98-0.85(\mathrm{~m}, 4 \mathrm{H}) 0.95(\mathrm{~d}, J=6.5 \mathrm{~Hz}, 3 \mathrm{H}), 0.94(\mathrm{~d}, J=6.4 \mathrm{~Hz}, 3 \mathrm{H}), 0.87(\mathrm{~d}, J=6.5 \mathrm{~Hz}, 3 \mathrm{H})$, $0.82(\mathrm{~d}, J=6.4 \mathrm{~Hz}, 3 \mathrm{H}), 0.81(\mathrm{~d}, J=6.4 \mathrm{~Hz}, 3 \mathrm{H}) .{ }^{13} \mathrm{C}-\mathrm{NMR}\left(100.6 \mathrm{MHz}, \mathrm{CDCl}_{3}\right): 199.29$ (s), 135.61 (d), $134.10(\mathrm{~s}), 129.44(\mathrm{~d}), 127.52(\mathrm{~d}), 68.67(\mathrm{t}), 50.94(\mathrm{t}), 45.51(\mathrm{t}), 45.22(\mathrm{t}), 44.42(\mathrm{t}), 41.11(\mathrm{t}), 33.20(\mathrm{~d})$, 28.67 (d), 27.66 (d), 27.47 (d), 27.34 (d), 26.89 (q), 23.26(t), 21.15 (q), 21.09 (q), 20.82 (q), 20.58 (q), 19.31 (s), 18.23 (q), 14.82 (q). $\mathrm{MS}(\mathrm{EI}+)$ for $\mathrm{C}_{35} \mathrm{H}_{56} \mathrm{O}_{2} \mathrm{SSi}: \mathrm{m} / \mathrm{z}(\%)=511(100 \%, \mathrm{M}-t$-Butyl), MS(CI+) for $\mathrm{C}_{35} \mathrm{H}_{56} \mathrm{O}_{2} \mathrm{SSi}: \mathrm{m} / \mathrm{z}(\%)=586\left(100 \%, \mathrm{M}+\mathrm{NH}_{4}^{+}\right)$. HRMS(EI+) for $\mathrm{C}_{35} \mathrm{H}_{56} \mathrm{O}_{2} \mathrm{SSi}: \mathrm{m} / \mathrm{z}(\%)=511(100 \%, \mathrm{M}-$ $t$-Butyl), Measured Mass: 511.3047 Da, Calculated Mass: 511.3066 Da.

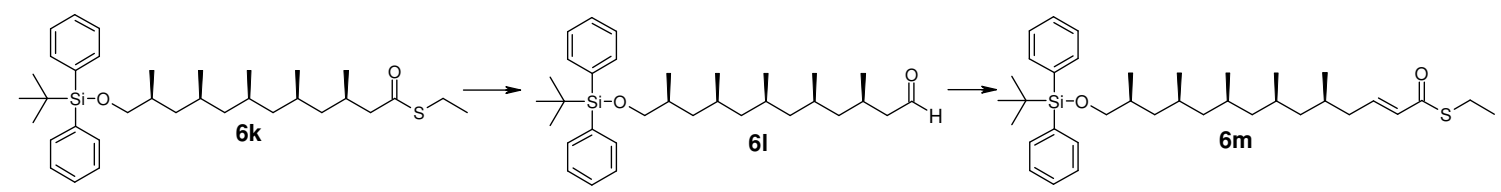

(-)-(5R,7R,9S,11S,13S)-14-(tert-Butyl-diphenyl-silanyloxy)-5,7,9,11,13-pentamethyl-tetradec-2enethioic acid S-ethyl ester (6m)

To a stirred mixture of $\mathbf{6 k}(512 \mathrm{mg}, 0.907 \mathrm{mmol})$ in THF $(20 \mathrm{~mL})$ was added 1.3 equiv of DIBALH ${ }^{4}(1 \mathrm{M}$ in toluene) $(1.180 \mathrm{~mL}, 1.180 \mathrm{mmol})$ at $-70{ }^{\circ} \mathrm{C}$ under nitrogen. Stirring was continued at $-70{ }^{\circ} \mathrm{C}$ until the reduction was completed (3-4 hour). The reaction mixture was poured into a saturated solution $(20 \mathrm{ml})$ of Rochelle salt (potassium sodium tartrate) and was stirred at $\mathrm{rt}$ for $30 \mathrm{~min}$. After phase separation and

\footnotetext{
${ }^{4}$ Reduction of the thioester with $\mathrm{Pd} / \mathrm{C}$ and $\mathrm{Et}_{3} \mathrm{SiH}$ once gave us the dithiane of the aldehyde as a side product. This problem was not observed with DIBALH.
} 
extraction of the aqueous phase with 3 portions of diethyl ether, the combined organic phases were dried over $\mathrm{MgSO}_{4}$, concentrated under reduced pressure and purified by flash chromatography (eluent pentane/ether 40:1) to afford aldehyde $\mathbf{6 l}$ which was used in the next step without complete removal of the eluent. A solution of aldehyde $\mathbf{6 l}$ and $\mathrm{Ph}_{3} \mathrm{PCHCOSEt}(430 \mathrm{mg}, 1.180 \mathrm{mmol})$ in $\mathrm{CH}_{2} \mathrm{Cl}_{2}(20 \mathrm{~mL})$ was heated at reflux for $24 \mathrm{~h}$. The solution was concentrated under reduced pressure and purified by flash chromatography (eluent pentane/ether 40:1) to afford $\alpha, \beta$-unsaturated thioester $\mathbf{6 m}$ as a colourless oil (372 $\mathrm{mg}, 69 \%$ yield over 2 steps, $\left.[\alpha]_{\mathrm{D}}=-6.8\left(c=1.21, \mathrm{CHCl}_{3}\right)\right)$. ${ }^{1} \mathrm{H}-\mathrm{NMR}\left(400 \mathrm{MHz}, \mathrm{CDCl}_{3}\right): \delta 7.68(\mathrm{dd}, J=$ 7.8, $1.6 \mathrm{~Hz}, 4 \mathrm{H}), 7.40(\mathrm{~m}, 6 \mathrm{H}), 6.88(\mathrm{dt}, J=15.4,8.0 \mathrm{~Hz}, 1 \mathrm{H}), 6.10(\mathrm{dt}, J=15.5,1.3 \mathrm{~Hz}, 1 \mathrm{H}), 3.52(\mathrm{dd}, J=$ 9.8, $5.0 \mathrm{~Hz}, 1 \mathrm{H}), 3.42(\mathrm{dd}, J=9.8,6.4 \mathrm{~Hz}, 1 \mathrm{H}), 2.95(\mathrm{q}, J=7.4 \mathrm{~Hz}, 2 \mathrm{H}), 2.24(\mathrm{~m}, 1 \mathrm{H}), 1.94(\mathrm{~m}, 1 \mathrm{H}), 1.74$ $(\mathrm{m}, 2 \mathrm{H}), 1.54(\mathrm{~m}, 3 \mathrm{H}), 1.39(\mathrm{~m}, 1 \mathrm{H}), 1.28(\mathrm{t}, J=7.4 \mathrm{~Hz}, 3 \mathrm{H}), 1.19(\mathrm{~m}, 4 \mathrm{H}), 1.06(\mathrm{~s}, 9 \mathrm{H}), 0.97-0.92(\mathrm{~m}$, $3 \mathrm{H}), 0.95(\mathrm{~d}, J=6.7 \mathrm{~Hz}, 3 \mathrm{H}), 0.89(\mathrm{~d}, J=6.6 \mathrm{~Hz}, 3 \mathrm{H}), 0.85(\mathrm{~d}, J=6.5 \mathrm{~Hz}, 3 \mathrm{H}), 0.82(\mathrm{~d}, J=6.5 \mathrm{~Hz}, 3 \mathrm{H})$, $0.81(\mathrm{~d}, J=6.5 \mathrm{~Hz}, 3 \mathrm{H}) .{ }^{13} \mathrm{C}-\mathrm{NMR}\left(100.6 \mathrm{MHz}, \mathrm{CDCl}_{3}\right): 189.95$ (s), 144.19 (d), 135.61 (d), 134.07 (s), $129.83(\mathrm{~d}), 129.42(\mathrm{~d}), 127.51(\mathrm{~d}), 68.64(\mathrm{t}), 45.47(\mathrm{t}), 45.24(\mathrm{t}), 44.37(\mathrm{t}), 41.10(\mathrm{t}), 38.97(\mathrm{t}), 33.16(\mathrm{~d})$, 29.93 (d), 27.62 (d), 27.42 (d), 27.34(d), 26.87 (q), 23.00 (t), 21.19 (q), 21.05 (q), 20.87 (q), 20.54 (q), 19.29 (s), 18.19 (q), 14.81 (q). MS(EI+) for $\mathrm{C}_{37} \mathrm{H}_{58} \mathrm{O}_{2} \mathrm{SSi}: \mathrm{m} / \mathrm{z}(\%)=537(100 \%, \mathrm{M}-t$-Butyl), MS(CI+) for $\mathrm{C}_{37} \mathrm{H}_{58} \mathrm{O}_{2} \mathrm{SSi}: \mathrm{m} / \mathrm{z}(\%)=612\left(100 \%, \mathrm{M}+\mathrm{NH}_{4}^{+}\right)$. HRMS(EI+) for $\mathrm{C}_{37} \mathrm{H}_{58} \mathrm{O}_{2} \mathrm{SSi}: \mathrm{m} / \mathrm{z}(\%)=537(100 \%, \mathrm{M}-$ $t$-Butyl), Measured Mass: 537.3198 Da, Calculated Mass: 537.3222 Da.

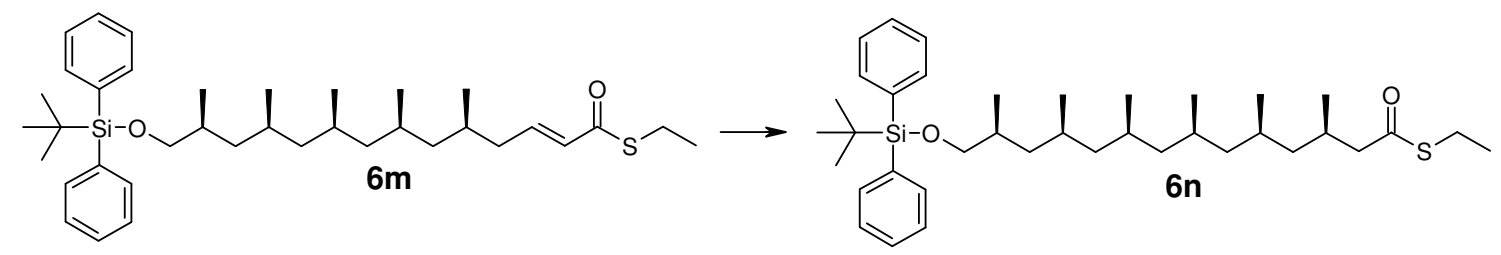

(-)-(3R,5R,7R,9S,11S,13S)-14-(tert-Butyl-diphenyl-silanyloxy)-3,5,7,9,11,13-hexamethyltetradecanethioic acid S-ethyl ester (6n)

$\left(R, S_{F e}\right)$-Josiphos $(4) \cdot \mathrm{CuBr}$ complex (3.1 mg, $\left.0.004 \mathrm{mmol}, 1 \mathrm{~mol} \%\right)$ was dissolved in $t$-BuOMe $(2.644 \mathrm{~mL})$ under nitrogen. The mixture was cooled to $-75^{\circ} \mathrm{C}$ and methylmagnesium bromide $(0.505 \mathrm{mmol}$, solution in diethyl ether) was added dropwise. After stirring for $10 \mathrm{~min}$, a solution of thioester (6m) (250 mg, 0.421 $\mathrm{mmol})$ in $t$-BuOMe $(0.731 \mathrm{~mL})$ was added via syringe pump over $1 \mathrm{~h}$. The reaction mixture was stirred at $75{ }^{\circ} \mathrm{C}$ for $17 \mathrm{~h}$, then quenched by the addition of $\mathrm{MeOH}$ and allowed to warm to room temperature. Saturated aqueous $\mathrm{NH}_{4} \mathrm{Cl}$ solution was then added. After phase separation and extraction of the aqueous phase with 3 portions of diethyl ether, the combined organic phases were dried over $\mathrm{MgSO}_{4}$, concentrated under reduced pressure and purified by flash chromatography (eluent pentane/ether 40:1) to afford $\mathbf{6 n}$ as a colourless oil $(242 \mathrm{mg}, 94 \%$ yield, syn/ant ratio by NMR $=>96 / 4$, d.r. calculated from syn/anti ratio $=$ $(3 R, 5 R, 7 R, 9 S, 11 S, 13 S):(3 S, 5 R, 7 R, 9 S, 11 S, 13 S):(3 R, 5 S, 7 R, 9 S, 11 S, 13 S):(3 R, 5 R, 7 S, 9 S, 11 S, 13 S):(3 R, 5 R, 7 S, 9 R$ $, 11 S, 13 S):(3 R, 5 R, 7 R, 9 S, 11 R, 13 S):(3 R, 5 R, 7 R, 9 S, 11 S, 13 R)=92: 1: 1: 1: 2: 2: 1$, diastereoisomers less than 0.04 were neglected. $\left.[\alpha]_{\mathrm{D}}=-4.92\left(c=1.10, \mathrm{CHCl}_{3}\right)\right)$. ${ }^{1} \mathrm{H}-\mathrm{NMR}\left(400 \mathrm{MHz}, \mathrm{CDCl}_{3}\right): \delta 7.68(\mathrm{dd}, J=7.8,1.6 \mathrm{~Hz}$, $4 \mathrm{H}), 7.40(\mathrm{~m}, 6 \mathrm{H}), 3.52(\mathrm{dd}, J=9.8,4.9 \mathrm{~Hz}, 1 \mathrm{H}), 3.42(\mathrm{dd}, J=9.8,6.4 \mathrm{~Hz}, 1 \mathrm{H}), 2.88(\mathrm{q}, J=7.4 \mathrm{~Hz}, 2 \mathrm{H})$, $2.56(\mathrm{dd}, J=14.3,5.0 \mathrm{~Hz}, 1 \mathrm{H}), 2.26(\mathrm{dd}, J=14.3,8.8 \mathrm{~Hz}, 1 \mathrm{H}), 2.12(\mathrm{~m}, 1 \mathrm{H}), 1.74(\mathrm{~m}, 1 \mathrm{H}), 1.54(\mathrm{~m}, 4 \mathrm{H})$, $1.40(\mathrm{~m}, 1 \mathrm{H}), 1.25(\mathrm{t}, J=7.4 \mathrm{~Hz}, 3 \mathrm{H}), 1.22(\mathrm{~m}, 4 \mathrm{H}), 1.06(\mathrm{~s}, 9 \mathrm{H}), 0.97-0.78(\mathrm{~m}, 5 \mathrm{H}) 0.95(\mathrm{~d}, J=6.6 \mathrm{~Hz}$, $3 \mathrm{H}), 0.94(\mathrm{~d}, J=6.6 \mathrm{~Hz}, 3 \mathrm{H}), 0.87(\mathrm{~d}, J=6.5 \mathrm{~Hz}, 3 \mathrm{H}), 0.84(\mathrm{~d}, J=7.0 \mathrm{~Hz}, 3 \mathrm{H}), 0.82(\mathrm{~d}, J=6.8 \mathrm{~Hz}, 3 \mathrm{H})$, $0.81(\mathrm{~d}, J=6.5 \mathrm{~Hz}, 3 \mathrm{H}) .{ }^{13} \mathrm{C}-\mathrm{NMR}\left(100.6 \mathrm{MHz}, \mathrm{CDCl}_{3}\right): 199.31$ (s), 135.62 (d), 134.08 (s), 129.43 (d), $127.52(\mathrm{~d}), 68.64(\mathrm{t}), 50.92(\mathrm{t}), 45.47(\mathrm{t}), 45.25(\mathrm{t}), 45.14(\mathrm{t}), 44.40(\mathrm{t}), 41.04(\mathrm{t}), 33.18(\mathrm{~d}), 28.67(\mathrm{~d}), 27.67$ (d), 27.49 (d), 27.43 (d), 27.35 (d), 26.88 (q), 23.26 (t), 21.29 (q), 21.23 (q), 21.13 (q), 20.82 (q), 20.57 (q), 19.29 (s), 18.22 (q), 14.81(q). MS(EI+) for $\mathrm{C}_{38} \mathrm{H}_{62} \mathrm{O}_{2} \mathrm{SSi}: \mathrm{m} / \mathrm{z}(\%)=553$ (100\%, M - $t$-Butyl), MS(CI+) for $\mathrm{C}_{38} \mathrm{H}_{62} \mathrm{O}_{2} \mathrm{SSi}: \mathrm{m} / \mathrm{z}(\%)=628\left(100 \%, \mathrm{M}+\mathrm{NH}_{4}^{+}\right)$. HRMS(EI+) for $\mathrm{C}_{38} \mathrm{H}_{62} \mathrm{O}_{2} \mathrm{SSi}: \mathrm{m} / \mathrm{z}(\%)=553(100 \%, \mathrm{M}-$ $t$-Butyl), Measured Mass: 553.3516 Da, Calculated Mass: 553.3535 Da. 


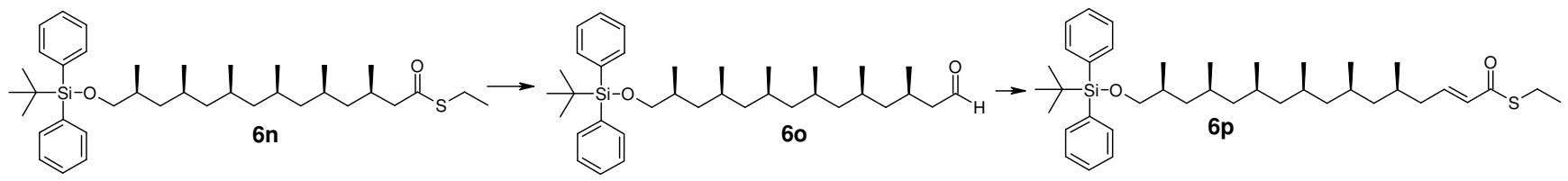

$(-)-(5 R, 7 R, 9 R, 11 S, 13 S, 15 S)-16$-(tert-Butyl-diphenyl-silanyloxy)-5,7,9,11,13,15-hexamethyl-hexadec2-enethioic acid $S$-ethyl ester (6p)

To a stirred mixture of $\mathbf{6 n}(236 \mathrm{mg}, 0.387 \mathrm{mmol})$ in $\mathrm{CH}_{2} \mathrm{Cl}_{2}(10 \mathrm{~mL})$ was added 1.3 equiv of DIBALH (1M in toluene) $(0.503 \mathrm{~mL}, 0.503 \mathrm{mmol})$ at $-70{ }^{\circ} \mathrm{C}$ under nitrogen. Stirring was continued at $-70{ }^{\circ} \mathrm{C}$ until the reduction was completed (3-4 hour). The reaction mixture was poured into a saturated solution (10 ml) of Rochelle salt (potassium sodium tartrate) and was stirred at $\mathrm{rt}$ for $30 \mathrm{~min}$. After phase separation and extraction of the aqueous phase with 3 portions of diethyl ether, the combined organic phases were dried over $\mathrm{MgSO}_{4}$, concentrated under reduced pressure and purified by flash chromatography (eluent pentane/ether 40:1) to afford aldehyde 60 which was used in the next step without complete removal of the eluent. A solution of aldehyde 60 and $\mathrm{Ph}_{3} \mathrm{PCHCOSEt}(183 \mathrm{mg}, 0.503 \mathrm{mmol})$ in $\mathrm{CH}_{2} \mathrm{Cl}_{2}(10 \mathrm{~mL})$ was refluxed for $24 \mathrm{~h}$. The solution was concentrated under reduced pressure and purified by flash chromatography (eluent pentane/ether 40:1) to afford $\alpha, \beta$-unsaturated thioester $\mathbf{6 p}$ as a colourless oil (193 mg, $78 \%$ yield over 2 steps, $\left.[\alpha]_{\mathrm{D}}=-4.70\left(c=0.72, \mathrm{CHCl}_{3}\right)\right) .{ }^{1} \mathrm{H}-\mathrm{NMR}\left(400 \mathrm{MHz}, \mathrm{CDCl}_{3}\right): \delta 7.67(\mathrm{dd}, J=$ 7.7, $1.5 \mathrm{~Hz}, 4 \mathrm{H}), 7.40(\mathrm{~m}, 6 \mathrm{H}), 6.87(\mathrm{dt}, J=15.4,7.1 \mathrm{~Hz}, 1 \mathrm{H}), 6.10(\mathrm{dt}, J=15.5,1.2 \mathrm{~Hz}, 1 \mathrm{H}), 3.52(\mathrm{dd}, J=$ 9.8, $5.0 \mathrm{~Hz}, 1 \mathrm{H}), 3.42(\mathrm{dd}, J=9.8,6.4 \mathrm{~Hz}, 1 \mathrm{H}), 2.94(\mathrm{q}, J=7.4 \mathrm{~Hz}, 2 \mathrm{H}), 2.23(\mathrm{~m}, 1 \mathrm{H}), 1.94(\mathrm{~m}, 1 \mathrm{H}), 1.73$ $(\mathrm{m}, 2 \mathrm{H}), 1.55(\mathrm{~m}, 4 \mathrm{H}), 1.39(\mathrm{~m}, 1 \mathrm{H}), 1.28(\mathrm{t}, J=7.4 \mathrm{~Hz}, 3 \mathrm{H}), 1.20(\mathrm{~m}, 4 \mathrm{H}), 1.06(\mathrm{~s}, 9 \mathrm{H}), 0.96-0.77(\mathrm{~m}$, $5 \mathrm{H}) 0.94(\mathrm{~d}, J=6.7 \mathrm{~Hz}, 3 \mathrm{H}), 0.89(\mathrm{~d}, J=6.6 \mathrm{~Hz}, 3 \mathrm{H}), 0.85(\mathrm{~d}, J=6.3 \mathrm{~Hz}, 3 \mathrm{H}), 0.84(\mathrm{~d}, J=6.4 \mathrm{~Hz}, 3 \mathrm{H})$, $0.82(\mathrm{~d}, J=6.6 \mathrm{~Hz}, 3 \mathrm{H}), 0.81(\mathrm{~d}, J=6.5 \mathrm{~Hz}, 3 \mathrm{H}) .0 .97-0.92(\mathrm{~m}, 5 \mathrm{H}) .{ }^{13} \mathrm{C}-\mathrm{NMR}\left(100.6 \mathrm{MHz} \mathrm{CDCl}_{3}\right)$ : $189.95(\mathrm{~s}), 144.17$ (d), 135.62 (d), $134.11(\mathrm{~s}), 129.86(\mathrm{~d}), 129.43$ (d), 127.52 (d), $68.68(\mathrm{t}), 45.51(\mathrm{t}), 45.28$ $(\mathrm{t}), 45.26(\mathrm{t}), 44.40(\mathrm{t}), 41.10(\mathrm{t}), 39.00(\mathrm{t}), 33.21(\mathrm{~d}), 29.97(\mathrm{~d}), 27.71(\mathrm{~d}), 27.52(\mathrm{~d}), 27.48(\mathrm{~d}), 27.43(\mathrm{~d})$, 26.89 (q), 23.01 (t), 21.31 (q), 21.30 (q), 21.12 (q), 20.90 (q), 20.56 (q), 19.30 (s), 18.21 (q), 14.82 (q). $\mathrm{MS}(\mathrm{EI}+)$ for $\mathrm{C}_{40} \mathrm{H}_{64} \mathrm{O}_{2} \mathrm{SSi}: \mathrm{m} / \mathrm{z}(\%)=579\left(100 \%, \mathrm{M}-t\right.$-Butyl), HRMS(EI+) for $\mathrm{C}_{40} \mathrm{H}_{64} \mathrm{O}_{2} \mathrm{SSi}: \mathrm{m} / \mathrm{z}(\%)=$ 579 (100\%, M - t-Butyl), Measured Mass: 579.3669 Da, Calculated Mass: 579.3692 Da.

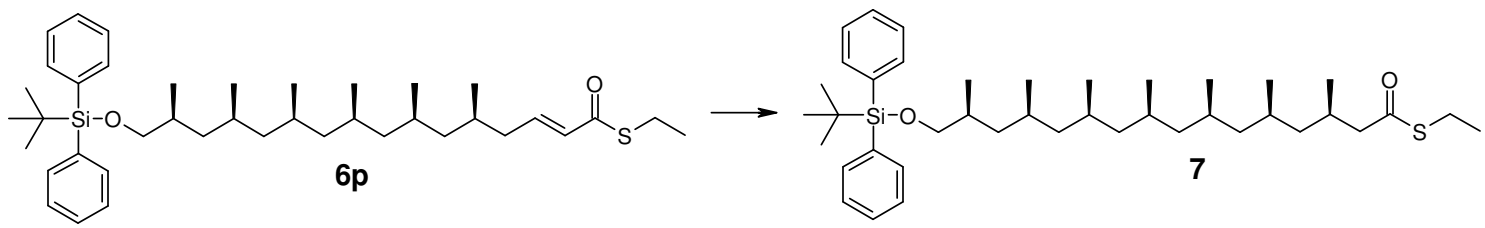

(-)-(3R,5R,7R,9S,11S,13S,15S)-16-(tert-Butyl-diphenyl-silanyloxy)-3,5,7,9,11,13,15-heptamethylhexadecanethioic acid $S$-ethyl ester (7)

$\left(R, S_{F e}\right)$-Josiphos $(4) \cdot \mathrm{CuBr}$ complex $(2.15 \mathrm{mg}, 0.003 \mathrm{mmol}, 1 \mathrm{~mol} \%)$ was dissolved in $t$-BuOMe $(1.820 \mathrm{~mL})$ under nitrogen. The mixture was cooled to $-75^{\circ} \mathrm{C}$ and methylmagnesium bromide $(0.349 \mathrm{mmol}$, solution in diethyl ether) was added dropwise. After stirring for $10 \mathrm{~min}$, a solution of thioester 6p (185 mg, 0.291 $\mathrm{mmol})$ in $t$-BuOMe $(0.500 \mathrm{~mL})$ was added via syringe pump over $1 \mathrm{~h}$. The reaction mixture was stirred at $75{ }^{\circ} \mathrm{C}$ for $17 \mathrm{~h}$, then quenched by the addition of $\mathrm{MeOH}$ and allowed to warm to room temperature. Saturated aqueous $\mathrm{NH}_{4} \mathrm{Cl}$ solution was then added. After phase separation and extraction of the aqueous phase with 3 portions of diethyl ether, the combined organic phases were dried over $\mathrm{MgSO}_{4}$, concentrated under reduced pressure and purified by flash chromatography (eluent pentane/ether 40:1) to afford 7 as a colourless oil $(175 \mathrm{mg}$, 92\% yield, syn/anti by $\mathrm{NMR}=>96 / 4$, d.r.calculated from syn/anti ratio $=$ $(3 R, 5 R, 7 R, 9 S, 11 S, 13 S, 15 S):(3 S, 5 R, 7 R, 9 S, 11 S, 13 S, 15 S):(3 R, 5 S, 7 R, 9 S, 11 S, 13 S, 15 S):(3 R, 5 R, 7 S, 9 S, 11 S, 13 S, 1$ $5 S):(3 R, 5 R, 7 R, 9 R, 11 S, 13 S, 15 S):(3 R, 5 R, 7 R, 9 R, 11 R, 13 S, 15 S):(3 R, 5 R, 7 R, 9 S, 11 S, 13 R, 15 S):(3 R, 5 R, 7 R, 9 S, 11 S$ $, 13 S, 15 R)=91: 1: 1: 1: 1: 2: 2: 1$, diastereoisomers less than 0.04 were neglected. $[\alpha]_{\mathrm{D}}=-5.50(c=1.05$, $\left.\mathrm{CHCl}_{3}\right)$ ). ${ }^{1} \mathrm{H}-\mathrm{NMR}\left(400 \mathrm{MHz}, \mathrm{CDCl}_{3}\right): \delta 7.68(\mathrm{dd}, J=7.8,1.6 \mathrm{~Hz}, 4 \mathrm{H}), 7.40(\mathrm{~m}, 6 \mathrm{H}), 3.53(\mathrm{dd}, J=14.8$, $9.8 \mathrm{~Hz}, 1 \mathrm{H}), 3.42(\mathrm{dd}, J=9.8,6.4 \mathrm{~Hz}, 1 \mathrm{H}), 2.88(\mathrm{q}, J=7.4 \mathrm{~Hz}, 2 \mathrm{H}), 2.56(\mathrm{dd}, J=14.3,5.0 \mathrm{~Hz}, 1 \mathrm{H}), 2.27$ 
(dd, $J=14.3,8.7 \mathrm{~Hz}, 1 \mathrm{H}), 2.12(\mathrm{~m}, 1 \mathrm{H}), 1.74(\mathrm{~m}, 1 \mathrm{H}), 1.55(\mathrm{~m}, 5 \mathrm{H}), 1.40(\mathrm{~m}, 1 \mathrm{H}), 1.25(\mathrm{t}, J=7.4 \mathrm{~Hz}, 3 \mathrm{H})$, $1.23(\mathrm{~m}, 5 \mathrm{H}), 1.06(\mathrm{~s}, 9 \mathrm{H}), 0.99-0.77(\mathrm{~m}, 6 \mathrm{H}), 0.95(\mathrm{~d}, J=6.6 \mathrm{~Hz}, 3 \mathrm{H}), 0.94(\mathrm{~d}, J=6.6 \mathrm{~Hz}, 3 \mathrm{H}), 0.87$ (d, $J=6.5 \mathrm{~Hz}, 3 \mathrm{H}), 0.84$ (broad d, $J=6.3 \mathrm{~Hz}, 6 \mathrm{H}), 0.82$ (broad d, $J=6.5 \mathrm{~Hz}, 6 \mathrm{H}) .{ }^{13} \mathrm{C}-\mathrm{NMR}(100.6 \mathrm{MHz}$, $\mathrm{CDCl}_{3}$ ): 199.27 (s), $135.62(\mathrm{~d}), 134.11(\mathrm{~s}), 129.43$ (d), $127.52(\mathrm{~d}), 68.69(\mathrm{t}), 50.96(\mathrm{t}), 45.48(\mathrm{t}), 45.29(\mathrm{t})$, $45.26(\mathrm{t}), 45.20(\mathrm{t}), 44.45(\mathrm{t}), 41.09(\mathrm{t}), 33.21$ (d), 28.68 (d), 27.72 (d), $27.54(3 \mathrm{x} \mathrm{C}, \mathrm{d}), 27.46$ (d), 26.89 (q), $23.26(\mathrm{t}), 21.41(\mathrm{q}), 21.36(\mathrm{q}), 21.26(\mathrm{q}), 21.15(\mathrm{q}), 20.84(\mathrm{q}), 20.57(\mathrm{q}), 19.30(\mathrm{~s}), 18.21$ (q), 14.81 (q). MS(EI+) for $\mathrm{C}_{41} \mathrm{H}_{68} \mathrm{O}_{2} \mathrm{SSi}: \mathrm{m} / \mathrm{z}(\%)=595\left(100 \%, \mathrm{M}-t\right.$-Butyl), HRMS(EI+) for $\mathrm{C}_{41} \mathrm{H}_{68} \mathrm{O}_{2} \mathrm{SSi}: \mathrm{m} / \mathrm{z}(\%)=$ 595 (100\%, M - t-Butyl), Measured Mass: 595.4006 Da, Calculated Mass: 595.4005 Da.

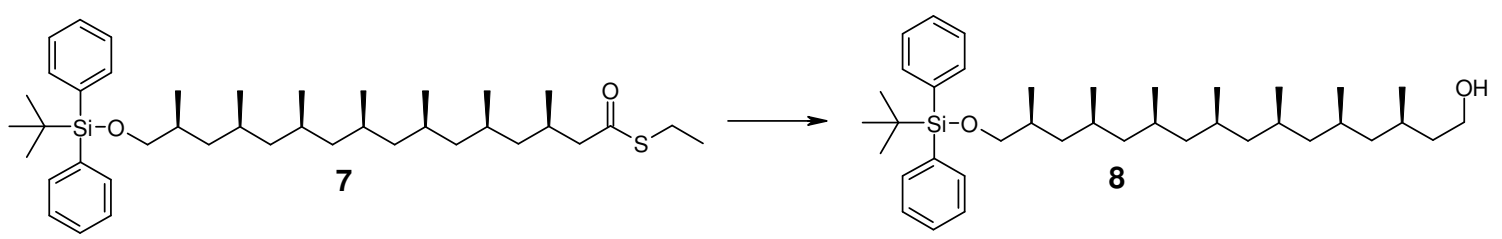

(-)-(3R,5R,7R,9S,11S,13S,15S)-16-(tert-Butyl-diphenyl-silanyloxy)-3,5,7,9,11,13,15-heptamethylhexadecan-1-ol (8)

To a stirred mixture of $7(91.0 \mathrm{mg}, 0.140 \mathrm{mmol})$ in $\mathrm{CH}_{2} \mathrm{Cl}_{2}(2 \mathrm{~mL})$ was added DIBALH $(0.182 \mathrm{~mL}, 0.182$ mmol, $1.0 \mathrm{M}$ solution in $\mathrm{CH}_{2} \mathrm{Cl}_{2}$ ) at $-50{ }^{\circ} \mathrm{C}$ under nitrogen. Stirring was continued until the reduction was completed (3-4 h). The reaction mixture was quenched in $5 \mathrm{~mL}$ saturated Rochelle solution (potassium sodium tartrate) and stirred for $30 \mathrm{~min}$. The phases were separated and the aqueous layer was extracted

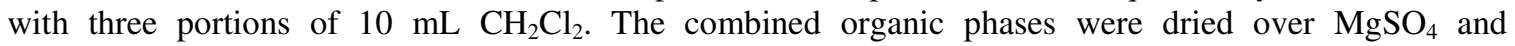
concentrated under reduced pressure to yield crude aldehyde. The above reduction/work-up was repeated to yield crude alcohol $\mathbf{8}$ as a colourless oil which was purified by flash chromatography (eluent pentane/ether $1: 1)$ to afford 8 as a colourless oil (76 mg, $91 \%$ yield, $\left.[\alpha]_{\mathrm{D}}=-4.70\left(c=0.86, \mathrm{CHCl}_{3}\right)\right)$. ${ }^{1} \mathrm{H}-\mathrm{NMR}(400 \mathrm{MHz}$, $\left.\mathrm{CDCl}_{3}\right): \delta 7.70(\mathrm{~d}, J=6.4 \mathrm{~Hz}, 4 \mathrm{H}), 7.41(\mathrm{~m}, 6 \mathrm{H}), 3.70(\mathrm{~m}, 2 \mathrm{H}), 3.54(\mathrm{dd}, J=9.78,5.0 \mathrm{~Hz}, 1 \mathrm{H}), 3.44(\mathrm{dd}, J$ $=9.7,6.4 \mathrm{~Hz}, 1 \mathrm{H}), 1.65(\mathrm{~m}, 7 \mathrm{H}), 1.43(\mathrm{~m}, 2 \mathrm{H}), 1.25(\mathrm{~m}, 7 \mathrm{H}), 1.08(\mathrm{~s}, 9 \mathrm{H}), 0.97(\mathrm{~d}, J=6.7 \mathrm{~Hz}, 3 \mathrm{H}), 0.94-$ $0.81(\mathrm{~m}, 6 \mathrm{H}), 0.92(\mathrm{~d}, J=6.5 \mathrm{~Hz}, 3 \mathrm{H}), 0.87(\mathrm{~d}, J=6.4 \mathrm{~Hz}, 3 \mathrm{H}), 0.87$ ( broad d, $J=6.5 \mathrm{~Hz}, 6 \mathrm{H}), 0.84$ (broad d, $J=6.5,6 \mathrm{H}) .{ }^{13} \mathrm{C}-\mathrm{NMR}\left(100.6 \mathrm{MHz}, \mathrm{CDCl}_{3}\right): 135.60$ (d), 134.07 (s), 129.42 (d), 127.51 (d), 68.64 $(\mathrm{t}), 61.19(\mathrm{t}), 45.47(\mathrm{t}), 45.32(\mathrm{t}), 45.26(2 \times \mathrm{C}, \mathrm{t}), 45.13(\mathrm{t}), 41.05(\mathrm{t}), 39.38(\mathrm{t}), 33.17(\mathrm{~d}), 27.68(\mathrm{~d}), 27.51(2$ x C, d), 27.46 (d), 27.41 (d), 26.91 (d), 26.88 (q), 21.43 (q), 21.35 (q), 21.32 (q), 21.14 (q), 20.99 (q), 20.56 (q), 19.28 (s), 18.21 (q). HRMS(EI+) for $\mathrm{C}_{39} \mathrm{H}_{66} \mathrm{O}_{2} \mathrm{Si}: \mathrm{m} / \mathrm{z}(\%)=537$ (18.2\%, M - $t$-Butyl), Measured Mass: 537.4112 Da, Calculated Mass: 537.4128 Da.

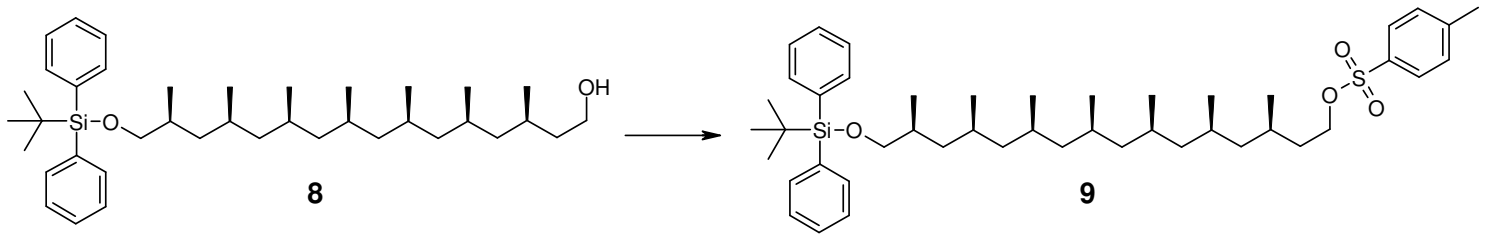

(-)-(3R,5R,7R,9S,11S,13S,15S)-Toluene-4-sulfonic acid 16-(tert-butyl-diphenyl-silanyloxy)$3,5,7,9,11,13,15$-heptamethyl-hexadecyl ester (9)

To a stirred mixture of $8(68 \mathrm{mg}, 0.114 \mathrm{mmol})$ and pyridine $(0.019 \mathrm{~mL}, 0.228 \mathrm{mmol})$ in $\mathrm{CH}_{2} \mathrm{Cl}_{2}(2 \mathrm{~mL})$ was added tosyl chloride $(44.8 \mathrm{mg}, 0.235 \mathrm{mmol}$ ) at rt under nitrogen, and the mixture was stirred for $24 \mathrm{~h}$. The reaction mixture was concentrated under reduced pressure and purified by flash chromatography (eluent pentane/ether 9:1) to afford 9 as a colourless oil $\left(84.4 \mathrm{mg}, 99 \%\right.$ yield, $\left.[\alpha]_{\mathrm{D}}=-4.00\left(c=0.40, \mathrm{CHCl}_{3}\right)\right) .{ }^{1} \mathrm{H}-$ NMR $\left(400 \mathrm{MHz}, \mathrm{CDCl}_{3}\right): \delta 7.81(\mathrm{~d}, J=8.4 \mathrm{~Hz}, 2 \mathrm{H}), 7.68(\mathrm{dd}, J=7.8,1.6 \mathrm{~Hz}, 4 \mathrm{H}), 7.39(\mathrm{~m}, 8 \mathrm{H}), 4.08(\mathrm{~m}$, 2H), $3.53(\mathrm{dd}, J=9.8,4.9 \mathrm{~Hz}, 1 \mathrm{H}), 3.43(\mathrm{dd}, J=9.7,6.4 \mathrm{~Hz}, 1 \mathrm{H}), 2.45(\mathrm{~s}, 3 \mathrm{H}), 1.74(\mathrm{~m}, 2 \mathrm{H}), 1.55(\mathrm{~m}, 4 \mathrm{H})$, $1.34(\mathrm{~m}, 4 \mathrm{H}), 1.17(\mathrm{~m}, 5 \mathrm{H}), 1.07(\mathrm{~s}, 9 \mathrm{H}), 0.95(\mathrm{~d}, J=6.7 \mathrm{~Hz}, 3 \mathrm{H}), 0.91-0.77(\mathrm{~m}, 24 \mathrm{H}) .{ }^{13} \mathrm{C}-\mathrm{NMR}(100.6$ $\mathrm{MHz}, \mathrm{CDCl}_{3}$ ): 144.54 (s), 135.59 (d), 134.05 (s), 133.24 (s), 129.75 (d), 129.41 (d), 127.83 (d), 127.50 (d), $69.07(\mathrm{t}), 68.63(\mathrm{t}), 45.43(\mathrm{t}), 45.21(\mathrm{t}), 45.15(\mathrm{t}), 45.09(\mathrm{t}), 44.61(\mathrm{t}), 41.03(\mathrm{t}), 35.14(\mathrm{t}), 33.16(\mathrm{~d}), 27.66$ (d), 27.47 (2 x C, d), 27.36 (d), 27.23 (d), 26.86 (q), 26.61 (d), 21.60 (q), 21.39 (q), 21.33 (q), 21.23 (q), 
21.12 (q), 20.83 (q), 20.00 (q), 19.28 (s), 18.19 (q). $\mathrm{HRMS}(\mathrm{EI}+)$ for $\mathrm{C}_{46} \mathrm{H}_{72} \mathrm{O}_{4} \mathrm{SSi}: \mathrm{m} / \mathrm{z}(\%)=691$ (3.6\%, M - $t$-Butyl), Measured Mass: 691.4190 Da, Calculated Mass: 691.4216 Da.

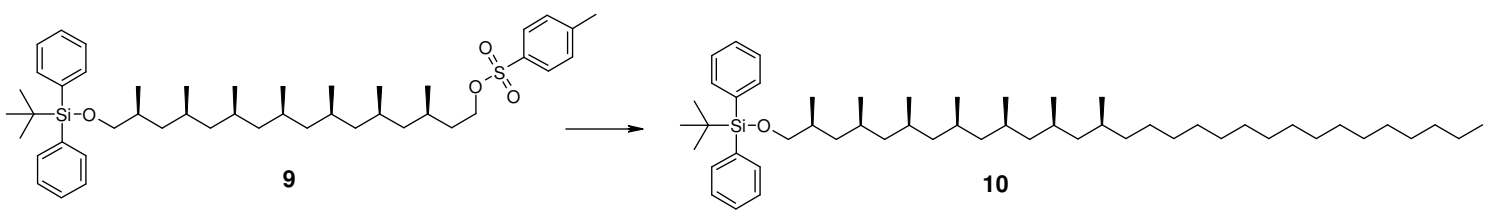

(-)-(2S,4S,6S,8S,10S,12S,14S)-tert-Butyl-(2,4,6,8,10,12,14-heptamethyl-triacontyloxy)-diphenyl-silane (10)

The Grignard reagent was freshly prepared as a $0.15 \mathrm{M}$ solution in THF. 1-Bromo-tetradecane $(152 \mathrm{mg}$, $0.548 \mathrm{mmol}$ ) was added to a stirred solution of activated (iodine) magnesium turnings ( $16 \mathrm{mg}, 0.658 \mathrm{mmol}$ ) and crushed glass in THF $(3.0 \mathrm{~mL})$. After $1 \mathrm{~h}$ at $45{ }^{\circ} \mathrm{C}$ the reaction mixture was cooled to $\mathrm{rt}$ and $0.5 \mathrm{ml}$ of the Grignard solution was titrated by using sec-BuOH and catalytic amounts of 1,10-phenanthroline. To a stirred mixture of $9(82 \mathrm{mg}, 0.110 \mathrm{mmol})$ and $\mathrm{CuBr} \cdot \mathrm{SMe}_{2}(4.5 \mathrm{mg}, 0.022 \mathrm{mmol}, 20 \mathrm{~mol} \%)$ in THF $(2 \mathrm{~mL})$ was added $\mathrm{C}_{18} \mathrm{H}_{37} \mathrm{MgBr}(2.200 \mathrm{~mL}, 0.330 \mathrm{mmol}, 0.15 \mathrm{M})$ at $0{ }^{\circ} \mathrm{C}$ under nitrogen. The reaction mixture was warmed to rt and stirred for $24 \mathrm{~h}$. After quenching with $2.0 \mathrm{~mL}$ of saturated $\mathrm{NH}_{4} \mathrm{Cl}$ solution, $5 \mathrm{ml}$ of diethyl ether was added. The phases were separated and the aqueous layer was extracted with three portions of 10 $\mathrm{mL}$ diethyl ether. The combined organic phases were dried over $\mathrm{MgSO}_{4}$ and concentrated under reduced pressure to yield crude 10, which was purified by flash chromatography (eluent pentane) to afford $\mathbf{1 0}$ as a colourless oil $\left(64 \mathrm{mg}, 75 \%\right.$ yield, $\left.[\alpha]_{\mathrm{D}}=-8.66\left(c=0.30, \mathrm{CHCl}_{3}\right)\right) .{ }^{1} \mathrm{H}-\mathrm{NMR}\left(400 \mathrm{MHz}, \mathrm{CDCl}_{3}\right): \delta 7.67(\mathrm{dd}$, $J=7.8,1.5 \mathrm{~Hz}, 4 \mathrm{H}), 7.40(\mathrm{~m}, 6 \mathrm{H}), 3.52(\mathrm{dd}, J=9.8,4.9 \mathrm{~Hz}, 1 \mathrm{H}), 3.41(\mathrm{dd}, J=9.8,6.5 \mathrm{~Hz}, 1 \mathrm{H}), 1.74$ $(\mathrm{m}, 1 \mathrm{H}), 1.51(\mathrm{~m}, 6 \mathrm{H}), 1.26(\mathrm{~m}, 32), 1.04(\mathrm{~s}, 9 \mathrm{H}), 0.94(\mathrm{~d}, J=6.7 \mathrm{~Hz}, 3 \mathrm{H}), 0.90-0.77(\mathrm{~m}, 24 \mathrm{H}) .{ }^{13} \mathrm{C}-\mathrm{NMR}$ (100.6 MHz, $\mathrm{CDCl}_{3}$ ): 135.62 (d), 134.10 (s), 129.43 (d), 127.52 (d), 68.68 (t), 45.50 (t), 45.47 (t), 45.33 (2 x C, t) 45.02 (t), 41.09 (t), 36.40 (t), 33.18 (d), $31.92(t), 30.05(t), 29.99$ (d), 29.75 (t), 29.70 (7 x C, t), $29.65(\mathrm{t}), 29.36(\mathrm{t}), 27.67(\mathrm{~d}), 27.54(2 \times \mathrm{C}, \mathrm{d}), 27.51(2 \times \mathrm{C}, \mathrm{d}), 26.88(\mathrm{q}), 22.68(\mathrm{t}), 21.42(\mathrm{q}), 21.36(\mathrm{q})$, 21.33 (q), 21.15 (q), 21.12 (q), 20.61 (q), 19.29 (s), 18.20 (q), 14.12 (t). HRMS(EI+) for $\mathrm{C}_{53} \mathrm{H}_{94} \mathrm{OSi}: \mathrm{m} / \mathrm{z}(\%)$ $=717(100 \%, \mathrm{M}-t$-Butyl), Measured Mass: 717.6346 Da, Calculated Mass: 717.6370 Da.

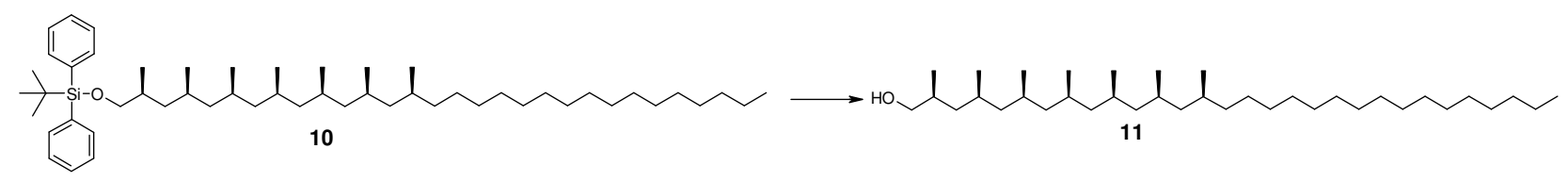

\section{(-)-(2S,4S,6S,8S,10S,12S,14S)-2,4,6,8,10,12,14-Heptamethyl-triacontan-1-ol (11)}

To a stirred mixture of $\mathbf{1 0}(20 \mathrm{mg}, 0.026 \mathrm{mmol})$ in THF $(2 \mathrm{~mL})$ at $\mathrm{rt}$ under nitrogen was added TBAF $(0.078 \mathrm{~mL}, 0.078 \mathrm{mmol}, 1.0 \mathrm{M}$ solution in THF), and the mixture was stirred for $5 \mathrm{~h}$. The reaction mixture was concentrated under reduced pressure and purified by chromatography (eluent pentane/ethanol 10:1) to afford 11 as a white solid with traces of siloxane. $\left(20 \mathrm{mg}\right.$, crude, $\left.[\alpha]_{\mathrm{D}}=-2.50\left(c=0.24, \mathrm{CHCl}_{3}\right)\right)$. ${ }^{1} \mathrm{H}-\mathrm{NMR}$ $\left(400 \mathrm{MHz}, \mathrm{CDCl}_{3}\right): \delta 3.55(\mathrm{dd}, J=10.3,4.9 \mathrm{~Hz}, 1 \mathrm{H}), 3.37(\mathrm{dd}, J=10.4,6.9 \mathrm{~Hz}, 1 \mathrm{H}), 1.72(\mathrm{~m}, 1 \mathrm{H}), 1.58$ $(\mathrm{m}, 3 \mathrm{H}), 1.51-1.12(\mathrm{~m}, 40 \mathrm{H}),(\mathrm{m}, 30 \mathrm{H})$. HRMS(EI+) for $\mathrm{C}_{37} \mathrm{H}_{76} \mathrm{O}: \mathrm{m} / \mathrm{z}(\%)=518\left(100 \%, \mathrm{M}-\mathrm{H}_{2} \mathrm{O}\right)$, Measured Mass: 518.5778 Da, Calculated Mass: 518.5790 Da.

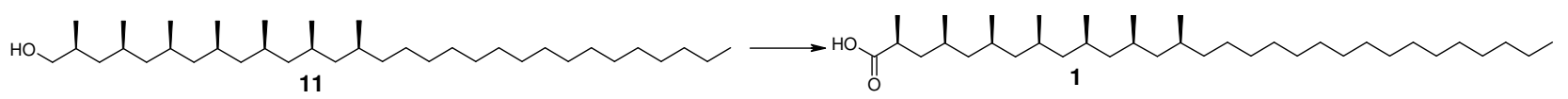


(+)-(2S,4S,6S,8S,10S,12S,14S)-2,4,6,8,10,12,14-Heptamethyl-triacontanoic acid (1)

To a stirred mixture of crude $11(20 \mathrm{mg}, 0.026 \mathrm{mmol})$ in $0.6 \mathrm{~mL} \mathrm{CCl}_{4}, 0.6 \mathrm{~mL} \mathrm{CH}_{3} \mathrm{CN}$ and $1.2 \mathrm{~mL} \mathrm{H}_{2} \mathrm{O}$ was added $\mathrm{RuCl}_{3} \cdot\left(\mathrm{H}_{2} \mathrm{O}\right)_{\mathrm{x}}(1.0 \mathrm{mg}, 0.005 \mathrm{mmol})$ and $\mathrm{NaIO}_{4}(24 \mathrm{mg}, 0.109 \mathrm{mmol})$ at $\mathrm{rt}$ under nitrogen. After $3 \mathrm{~h}$ the reaction mixture was poured in $2 \mathrm{~mL} \mathrm{CH}_{2} \mathrm{Cl}_{2}$ and $0.5 \mathrm{~mL}$ water was added. The phases were separated and the aqueous layer was extracted with three portions of $5 \mathrm{~mL} \mathrm{CH}_{2} \mathrm{Cl}_{2}$. The combined organic phases were dried over $\mathrm{MgSO}_{4}$ and concentrated under reduced pressure to yield crude 1, which was purified by flash chromatography (eluent pentane/diethyl ether 9:1) to afford 1 as a colourless oil (13 mg, $90 \%$ yield. The product did not contain other diasteroisomers according to ${ }^{13} \mathrm{C}-\mathrm{NMR}$, most probably due to chromatography steps. $[\alpha]_{\mathrm{D}}=+4.60\left(c=1.12, \mathrm{CHCl}_{3}\right),{ }^{1} \mathrm{H}-\mathrm{NMR}\left(400 \mathrm{MHz}, \mathrm{CDCl}_{3}\right): \delta 2.59(\mathrm{~m}, 1 \mathrm{H}), 1.77$ $(\mathrm{m}, 1 \mathrm{H}), 1.57(\mathrm{~m}, 4 \mathrm{H}), 1.50-0.94($ broad m, 34H) $1.19(\mathrm{~d}, J=6.9 \mathrm{~Hz}, 3 \mathrm{H}), 1.10-0.96(\mathrm{~m}, 4 \mathrm{H}), 0.94-$ 0.79, 27H). ). ${ }^{13} \mathrm{C}-\mathrm{NMR}\left(100.6 \mathrm{MHz}, \mathrm{CDCl}_{3}\right): 182.55(\mathrm{~s}), 45.48(\mathrm{t}), 45.42(2 \times \mathrm{C}, \mathrm{t}), 45.08(\mathrm{t}), 45.05(\mathrm{t})$, $40.72(\mathrm{t}), 37.16(\mathrm{~d}), 36.43(\mathrm{t}), 31.92(\mathrm{t}), 30.05(\mathrm{t}), 29.98(\mathrm{~d}), 29.75(\mathrm{t}), 29.69(7 \times \mathrm{x}, \mathrm{t}), 29.65(\mathrm{t}), 29.35(\mathrm{t})$, 28.16 (d), 27.48 (2 x C, d), 27.43 (d), 27.24 (d), $26.85(\mathrm{t}), 22.68(\mathrm{t}), 21.28(\mathrm{q}), 21.21$ (q), 21.12 (q), 20.85 (q), 20.59 (q), 20.54 (q), 18.13 (q), 14.11 (q). HRMS(EI+) for $\mathrm{C}_{37} \mathrm{H}_{74} \mathrm{O}_{2}: \mathrm{m} / \mathrm{z}(\%)=550$ (100\%), Measured Mass: $550.5682 \mathrm{Da}$, Calculated Mass: $550.5689 \mathrm{Da}$. See spectral supporting info for fragmentation details.

A small sample $(5.4 \mathrm{mg})$ was converted into the methyl ester of $\mathbf{1}$ (treatment with trimethylsilyldiazomethane) for optical rotation and mass analysis to compare with literature values: Goren, M. B.; Brokl, O.; Das, B. C.; Lederer, E. Biochemistry 1971, 10, 72-81.

${ }^{1} \mathrm{H}-\mathrm{NMR}\left(400 \mathrm{MHz}, \mathrm{CDCl}_{3}\right): \delta 3.66(\mathrm{~s}, 3 \mathrm{H}), 2.58(\mathrm{~m}, 1 \mathrm{H}), 1.77-1.39$ (broad m, 5H), 1.30 - 1.08 (broad m, $38 \mathrm{H}), 1.05-0.74$ (broad m, 27H).

MS(EI+) for $\mathrm{C}_{38} \mathrm{H}_{78} \mathrm{O}_{2}: \mathrm{m} / \mathrm{z}(\%)=564(77 \%, M)$. See spectral supporting info for fragmentation details. Mass analysis matched with literature.

Measured optical rotation: $[\alpha]_{\mathrm{D}}=+6.2\left(c=0.55, \mathrm{CHCl}_{3}\right)$, literature value for mixture of homologues of phthioceranic methyl ester: $[\alpha]_{\mathrm{D}}=+7.9\left(c=2.03, \mathrm{CHCl}_{3}.\right)$ 